\title{
Non-linear mechanics in resonant inertial micro sensors
}

\author{
Claudia Comi ${ }^{\mathrm{a}}$, Valentina Zega ${ }^{\mathrm{a}}$, Alberto Corigliano $^{\mathrm{a}, 1}$ \\ ${ }^{a}$ Department of Civil and Environmental Engineering, Politecnico di Milano, Italy
}

\begin{abstract}
Microsystems (or Micro Electro Mechanical Systems, MEMS) are important components of many popular products in the consumer market and are one of the enabling ingredients of incoming industrial revolutions like Industry 4.0 and Internet of Things (IoT). Behind many Microsystems there are important mechanical principles and coupling effects that must be completely mastered starting from the design phase. More sophisticated and smaller devices also imply to consider many non-linear effects that can be strictly related to the mechanical response or to coupled electro-thermo-mechanical phenomena. This paper contains an overview of key mechanical aspects in design and reliability of Microsystems with a particular focus on non-linear dynamics of oscillators in inertial sensors.
\end{abstract}

Key words: Microsystems, MEMS, Multi-physics, mechanical oscillators, resonators, non-linear dynamics

2010 MSC: 00-01, 99-00

\section{Introduction}

Microsystems (or Micro Electro Mechanical Systems, MEMS) are complex devices conceived combining multi-physics principles and produced with technologies similar to the ones used for integrated circuits, in which smaller dimensions can be in the order of fractions of micrometers [1, 2, 3, 4, 5, 6, 7, 8]. Microsystems technology can nowadays be used to produce highly miniaturized

\footnotetext{
* Corresponding author

Email address: alberto.corigliano@polimi.it (Alberto Corigliano)
}

Preprint submitted to Journal of ${ }^{A} T_{E} X$ Templates

October 26, 2019 
sensors such as accelerometers $[9,10,11]$, gyroscopes [3, 12], Lorentz force magnetometers $[13,14,15]$ and pressure sensors $[16,17,18]$, or actuators which in turn can be used to create e.g. micro-mirrors [19, 20, 21, 22, 23, 24], micro ultrasound transducers [25, 26], micro-pumps [27], gas sensors [28], resonators $[29,30,31,32]$ or energy harvesting devices [33, 34, 35, 36, 37, 38, 39].

The great versatility and the reduced unit cost have been the basic ingredients for the large and fast diffusion of microsystems. These are nowadays complex products of modern engineering which find a large number of applications in various fields such as consumer market, automotive engineering, structural monitoring and biomedical engineering. MEMS are one of the enabling ingredients of the incoming revolutions of Internet of Things (IoT) and Industry 4.0 due to the possibility to sense and actuate by means of many, very small objects $[37,40,41]$.

Mechanical issues, and particularly mechanical reliability, are extremely important in all the phases of MEMS design and in the development of relevant production technology. Proper design and fabrication of micro-devices must ensure the perfect functioning both in common exercise conditions and in extreme situations (e.g. accidental drop, mechanical and electrical shock, harsh environment, etc.). Complex non-linear dynamic responses of the oscillators can be induced e.g. by the electro-mechanical coupling due to electrostatic actuation, by the interaction between membrane and bending regimes in slender beams or plates, by the temperature variations, by the internal contacts between surfaces at low distance, [2]. All non-linear phenomena related to the mechanics of microsystems must be well understood to obtain effective designs that can be transformed into real products. In most cases linear response is required to simplify the read-out control circuit and to obtain predictable devices with low dispersion in the final properties. To achieve this requirement and in particular to eliminate the effect of chaos, in [42], an adaptive dynamic 35 surface control scheme with extended state observer is designed to convert random motion into regular motion without precise system model parameters and measured variables. In [43] and [44] the shape optimization is exploited to tai- 
lor the non-linear response of MEMS resonators and resonant accelerometers, respectively. In some cases non-linear regimes cannot be avoided due to the physics of the problems. In other situations non-linear regimes can also be advantageous to obtain peculiar responses. In [45], for example, the non-linear behavior of a MEMS clamped-clamped beam is exploited to realize a switch triggered by gas, while in [46], a new MEMS gas sensor based on the dynamicbifurcation detection technique is proposed. In [47] a threshold shock sensor based on a bistable mechanism is proposed, while in [48] the non-linear dynamics of MEMS resonators, specifically, bi-stability and hysteresis, is used to simulate the detection and memory of a single rate model neuron, thus providing a novel concept for a micro-electro-mechanical-system (MEMS) neural computing unit. In [49], a band-pass filter is obtained by combining the non-linear softening, hardening, and veering phenomena (near crossing) of two vibration modes of a MEMS resonator that get close to each others, while in [50] novel threshold pressure sensors based on non-linear dynamics of MEMS resonators are presented. Finally, in [51] the non-linear regime is employed to improve the sensitivity of a MEMS gyroscope.

The purpose of this review paper is to describe some aspects of the complexity of microsystems from a mechanical perspective with particular reference to non-linear mechanical problems in MEMS inertial sensors focusing on real devices designed and studied by the Authors.

The paper is organized as follows.

In Section 2 a general discussion on possible sources of nonlinearities in microsystems is proposed, mentioning in particular dissipative phenomena which are the origin of damping, fracture, fatigue, spontaneous adhesion or stiction, mechanical non-linear effects induced by large strains and displacements, electromechanical coupling.

65 Section 3 focuses on mechanical oscillators in MEMS. After general remarks, it is shown how vibrating systems, like masses suspended through beams, can be reduced to a 1 degree of freedom (dof) equivalent oscillator which is governed by a general non-linear 2nd order differential equation in time. Among the various 
non-linear oscillators, the Duffing one is taken as a meaningful example. The Section is closed mentioning the important issue of parametric resonance which is described by the Mathieu equation and can emerge in various situations alone or in combination with other non-linear effects.

Sections from 4 to 8 contain practical examples, taken from the Authors' recent experience, of the importance of non-linear dynamic responses in MEMS.

75 Section 4 concerns the hardening and softening responses which can be observed in a L-shaped oscillator designed for a uniaxial resonant accelerometer. Section 5 discusses the non-linear response of a Double Ended Tuning Fork Resonator designed for a resonant accelerometer in the presence of temperature variations. Section 6 describes a torsional resonator and its softening behavior induced by electrostatic actuation and readout. Section 7 concerns peculiar non-linear responses obtained with elastic connections used in Coriolis-based vibrating gyroscopes. The parametric resonance of a disk ring gyroscope is discussed in the last example of Section 8 .

Closing remarks and perspectives are included in the final Section 9.

\section{Sources of non-linear responses in the dynamics of MEMS}

The dynamic behavior of a MEMS or of an oscillating part of it, after proper simplifications, can be described (see [1]) by the relative dynamic equilibrium equation of a pointwise mass $m$

$$
m \mathbf{a}=-m \mathbf{a}_{0}-m \mathbf{a}_{c}+\mathbf{F}
$$

where $\mathbf{a}$ is the acceleration in the non-inertial reference frame of the MEMS, $\mathbf{a}_{0}$ is the external acceleration, $\mathbf{a}_{c}$ is the Coriolis's acceleration and $\mathbf{F}$ is the total force. This latter has usually three contributions: an internal force due to the reactions of the deformable elements which suspend the mass (also referred to as springs), a force accounting for damping effects and a driving term, often due to electrostatic actuation. 
The non-linear dependence on displacement and/or velocity of each one of the above forces leads to a dynamic non-linear behavior of the MEMS device (or component).

\subsection{Internal forces}

The internal reactions of deformable bodies in MEMS depend on displacements, deformations and stresses to which deformable parts are subject. Stresses are usually low with respect to the elastic limit of the material during the regular working conditions and the mechanical behavior can then be considered as linear elastic [52]. This situation changes when the device is subject to non-common working conditions like e.g. in the cases of accidental impacts, when the stress levels can provoque fracture. Fracture phenomena always imply a non-linear response due to the variation of instantaneous material and structural stiffness related to the propagation of cracks. Accidental drop events and fracture in microsystems have been extensively studied e.g. in $[53,54,55,56,57]$, they represent an highly non-linear mechanical response in MEMS, that must be studied and governed with the approaches of Fracture Mechanics.

In many cases, as discussed in the subsequent Sections, inside MEMS there are oscillating parts which are kept in motion by means of on-board actuators (see e.g. [58]). The frequency of oscillations can be high, in the order of tens of thousands of Hertz like in micro-gyroscopes or even very high, in the order of millions of Hertz, like in resonators; this means that during the expected lifetime the oscillating part will undergo billions of cycles. This situation can imply problems related to mechanical fatigue or subcritical crack propagation, a non-linear phenomenon well studied in metallurgy which appears for stress levels which are much lower than those able to provoque instantaneous fracture. Fatigue failure in MEMS has been studied and experimental evidences show that the phenomenon can appear also in silicon devices, usually for stress levels that are much higher than those used in regular working conditions for oscillating parts and for very high number of cycles (see e.g. [59, 60, 61, 62]).

The internal reactions of deformable bodies can depend non-linearly on the 
level of displacement and deformation also in the linear elastic regime, due to socalled non-linear geometric effects. As a meaningful example, when the flexural oscillations of a beam are small, the bending behavior is completely decoupled from the axial one and the study of the deformable body can be carried out in the geometrically linear regime. In some meaningful cases, as those discussed in the Sections 4, 5 and 7, the axial response affects the bending one, then a geometrically nonlinear response is activated with the bending stiffness dependent on the level of axial force in the beam. Depending on the structural configuration, this effect can induce so-called mechanical hardening or softening with interesting implications on the non-linear dynamic response (see e.g. [63, 64]). Geometric nonlinearities in MEMS usually end-up in an hardening behavior of the mechanical structure, however, in the literature there are examples of microstructures that exhibit softening mechanical behaviors [65, 66, 67, 68, 69].

\subsection{Damping effects}

Damping is usually represented as an equivalent viscous contribution, i.e. by a force linearly dependent on the velocity; in reality, damping in MEMS originates from many different sources, [1].

An important source, called fluid damping [70, 71], comes from the fluidstructure interaction of solid portions moving at high frequency inside MEMS boxes in which gas at various pressures is contained. This happens e.g. in resonant accelerometers and in the majority of micro-gyroscopes. The quantitative evaluation of fluid damping must be based on the accurate representation of the fluid-structure interaction at varying internal pressures. Different regimes must be distinguished for the fluid which go from the standard fluid dynamics represented by Navier-Stokes equations, which hold at atmospheric pressure, to rarefied gas dynamics with non-deterministic, statistical descriptions like Boltzmann equation, which hold at low pressure. In peculiar situations, like e.g. for the movement of micro-mirrors plates, it is not possible to simplify the fluid damping with linear terms and non-linear effects must be considered [72, 73].

When the pressure inside the MEMS box reduces to very low values, near 
to vacuum conditions, fluid damping becomes negligible, while an important source of damping comes from the interaction of thermal and mechanical fields in the thermoelastic responses. This form of solid damping, called thermoelastic damping or TED $[74,75,76,77]$ cannot be easily eliminated, it can be only reduced by careful design of the deformable portions: reducing the volumetric part of the deformation makes them less prone to thermoelastic damping [78, 79].

Another important component of solid damping in microsystems is represented by the transformation of kinetic energy of vibrating parts in energy carried by waves that are dispersed in the substrate; this phenomenon typically occurs at the anchor points and is referred to as anchor losses [80].

Other sources of solid damping are present due to e.g. internal rearrangements of crystals in polycrystalline materials or to the scattering of the acoustic phonons associated with the resonant mode with thermal phonons both in the Landau-Rumer regime and the Akhiezer regime [81].

In general terms, damping phenomena involving vibrating parts in microsystems are very complex and must be well understood for every specific device to be designed.

\subsection{Electrostatic forces}

Often MEMS are electrostatically actuated and/or sensed and the electrostatic forces are a typical source of nonlinearity in the mechanical response of MEMS. Consider for instance the case where the driving force is generated by a parallel plate configuration through a potential difference between the movable part of the MEMS (shuttle) kept at voltage $V=V_{p}$ and fixed electrodes kept at voltage $V=0$ as depicted in Fig. 1.

The electrostatic force non-linearly depends on the displacement $u$ of the shuttle

$$
F^{e l e c}=\frac{1}{2} \epsilon_{r} \epsilon_{0} S V_{p}^{2} \frac{1}{\left(g_{0}-u\right)^{2}},
$$

where $\epsilon_{r}$ and $\epsilon_{0}$ are the relative and absolute permittivity constants, $S$ is the surface of the plates and $g_{0}$ is the relative initial distance between the plates. 


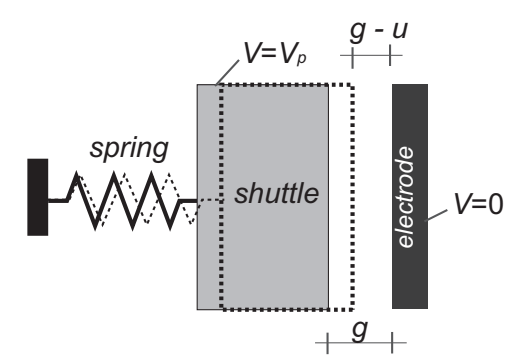

Figure 1: Scheme of the parallel plate driving

The first order linear approximation around the static solution $u=u_{p}$ of eq. (2) leads to the definition of the equivalent electrostatic stiffness $k_{e}$ which adds a negative contribution to the mechanical linear stiffness

$$
k_{e}=-\frac{1}{g^{3}} \epsilon_{r} \epsilon_{0} S V_{p}^{2}
$$

where $g=g_{0}-u_{p}$. At increasing voltage, or decreasing gap between parallel plates, the stiffness of the system reduces, and the so-called electrostatic softening effect appears, as discussed in the examples of Sections 4, 6 and 7.

Some recent MEMS designs exploit the effect of fringing fields electrostatic forces. At difference from parallel plate actuators, in fringing fields actuators the electrostatic forces can produce both an increase and a decrease of the equivalent stiffness. In [82] this effect was first studied and experimentally demonstrated. As discussed in [83], with a suitable design of side electrodes, these forces have a restoring effect on a vibrating cantilever and thus induce an increase of the apparent stiffness, causing the so-called electrostatic hardening. Furthermore, it is shown that combining the tuning of fringing fields and parallel plates potentials can lead to peculiar non-linear behavior, such as bi-stable behavior.

\subsection{Low distance and contact forces}

Due to the small dimensions of all gaps between the moving and fixed parts in MEMS and to accidental impacts, several surfaces may come at very low dis- 
tance or even at contact. These events can induce an additional source of nonlinearity in the microsystems response, due to the possibility that the surfaces will remain adhered after contact. This phenomenon, called stiction, is highly non-linear and is usually considered as a serious reliability issue in MEMS. Stiction is governed by forces that arise at very narrow distances between surfaces. In the presence of humidity, the main attractive forces come from capillary attraction, while in dry conditions the always present Casimir/Polder and van der Waals forces govern the phenomenon (see $[84,85,86]$ ).

Strictly linked to contact and stiction there are other sources of nonlinearities coming from repeated contacts of surfaces of oscillating parts against fixed or other movable portions. These are difficult to control and can transform in complex dynamic responses. In [87], for example, a frequency comb induced by contact dynamics in MEMS is put in evidence both numerically and experimentally, while in [88] an assessment of the Newmark method is proposed to compute chaotic vibrations of impacting oscillators. In the same field, a theoretical study of a very complex scenario of a bilinear oscillator, with a rich structured pattern that includes continuous and discontinuous bifurcations, flip bifurcations and alternating regular and chaotic regimes is proposed in [89], while in [90] the response and stability of piecewise linear oscillators under multi-forcing frequencies are investigated.

\subsection{Thermal effects}

As already mentioned when discussing the TED, thermal phenomena can have an important influence on the mechanical response of microsystems. In general, temperature always influences the mechanical response through the presence of thermal strains and through the mismatch of the coefficient of thermal expansion (CTE) of the various materials composing the devices. Moreover, material parameters like Young's modulus and CTE themselves change with temperature $[91,92]$ thus deeply influencing the frequency response of the MEMS resonant devices.

The temperature dependence of the resonant frequency of MEMS devices has 
been extensively studied in the literature especially in resonators for real time clocking applications $[79,93,94]$. In particular, the temperature dependence of the natural frequency has been investigated in combination with nonlinearities, as in the example of Section 5. In [95], for example, the authors exploit nonlinearities to compensate the frequency variation in temperature of a MEMS gyroscope.

CTEs mismatch and variable temperature conditions during fabrication can be the source of residual stresses at the end of the fabrication process, which in turn can produce non-linear effects in the dynamic response of oscillating beams and plates (see chapter 14 in [1] and references therein). This source of nonlinearity is strictly process-related and in many cases very difficult to dominate with simple modelling.

\subsection{Other non-linear dynamic phenomena}

When the resonance frequencies of distinct modes of the MEMS oscillator satisfy a commensurate relationship, strong coupling and energy transfer among them can happen [96, 97]: this phenomenon is usually referred to as internal resonance. In [98], for example, a novel response of a non-linear micromechanical resonator when operated in a region of strong, non-linear mode coupling is demonstrated: the system is excited with a single drive signal and its response is characterized by periodic amplitude modulations that occur at timescales based on system parameters. In [99] it is shown that it is possible 255 to stabilize the oscillation frequency of non-linear self-sustaining micromechanical resonators by coupling two different vibrational modes through an internal resonance, while in [100] the two-to-one internal resonance between the first two symmetric vibrational modes of a micromachined arch resonator is theoretically and experimentally investigated. Sarrafan et al. in [101], describe and experimentally demonstrate the potential for employing internal resonance for detection of angular rate signals, where the Coriolis effect modifes the energy coupling between the distinct drive and sense vibration modes. 
Chaos is another type of non-linear motion which is usually undesirable in the dynamic response of many systems; however, it has been proven to be useful at numerous applications such as sensing, fluid mixtures and secure communication $[102,103]$. Due to desirable and undesirable effects of chaos on the performance of the micro and nano resonators, it is necessary to predict the occurrence of this phenomenon in the dynamic response of the systems and several studies have been devoted to the investigation of chaos in nano resonators $270[104,105]$.

Another phenomenon that can be induced by nonlinearities in MEMS oscillators is the synchronization [106].

The above mentioned sources of non-linear behaviors are meaningful but do not cover all the complexity of non-linear mechanics in MEMS. Other sources of nonlinearities can be present, in particular due to other multi-physics phenomena, like e.g. in the presence of magnetic fields.

\section{Mechanical oscillators as key component in MEMS}

Mechanical oscillators are typical components of many microsystems; they are usually created on board by complex geometries of deformable beams and

plates. However, in many cases, their dynamic behavior can be described by an equivalent 1 dof or 2 dof non-linear oscillator. This, in turn contains the main sources of non-linear dynamic behaviors discussed in the subsequent Sections $4-8$.

\subsection{From continuum to discrete formulation}

285

The dynamic equilibrium equations of a deformable body can be obtained making use of the Hamilton's variational principle (see [107] for more details).

A discrete formulation is obtained by approximating the displacement field $\mathbf{u}=\mathbf{u}(\mathbf{x}, t)$ as:

$$
\mathbf{u}(\mathbf{x}, t)=\sum_{i=1}^{n} \boldsymbol{\Psi}_{i}(\mathbf{x}) U_{i}(t)
$$


By retaining only the first $m$ terms in (4), one reduces the dynamic behavior We focus now on systems such that just 1 dof can be retained $\left(U_{1}=U\right)$. The non-linear dependence on $U$ is often transformed in polynomial expressions after development in Taylor series expansion of the non-linear functions. The equation of motion can thus be expressed as follows,

$$
m \ddot{U}+b \dot{U}+\sum_{j=1}^{p} k_{j} U^{j}=F(t) .
$$

The linear case corresponds to $p=1$. The expressions of the equivalent mass $m$, damping coefficient $b$, stiffnesses $k_{j}$ and force $F$ depend on the specific problem considered and will be given in the following Sections for some meaningful cases. In general, $F(t)$ contains the external forces, if any, and the actuation forces. Considering electrostatic actuation, the driving force depends on the square of the potential, which usually has a constant DC term $V_{p}$ and an oscillating $\mathrm{AC}$ term $v_{a}(t)$. As discussed e.g. in [108], this introduces a forcing term with a linear dependence on $v_{a}(t)$ and a non-linear forcing term. This latter can be neglected if the amplitude of the oscillating voltage is small with respect to the $\mathrm{DC}$ voltage, as it happens in the devices described in the following sections.

Often, for moderate values of the DC potential, one can arrest the polynomial terms in (5) to the third order and, when the non-linear second order term $k_{2} U^{2}$ is negligible with respect to the third order one, by setting $k_{2}=0$, one obtains the so-called Duffing oscillator, with the peculiar dynamic behavior recalled in the next section. Note that cubic (and possibly quadratic) terms in the Duffing oscillator equations arise from geometric or electrostatic nonlinearities.

For other problems, more terms should be considered in the Taylor expansion, as shown e.g. in the example of Section 6. The interested reader can refer to the review recently published by Tiwari et al. [109].

When two or more dof are retained in Eq. 4, a system of non-linear equations 15 is obtained, as for the disk ring gyroscope discussed in Section 8. 


\subsection{Duffing oscillator}

The third order Duffing oscillator is an example of non-linear dynamics in MEMS that can be found in many applications. The solution of eq. (5), with $p=3$ and $k_{2}=0$, can be obtained from series of successive approximations as,

$$
U=\bar{U} \cos (\omega t)-\frac{\bar{U}^{3}}{32} \frac{k_{3}}{k_{1}} \cos (3 \omega t) .
$$

In eq. (6) $\bar{U}$ is the oscillation amplitude and $\omega$ is the actual value of the angular frequency which differs from the reference value of the linear case $\omega_{0}$ :

$$
\omega_{0}=2 \pi f_{0}=\sqrt{\frac{k_{1}}{m}} .
$$

Under the assumption of very high $Q$-factor, $\omega$ is expressed as:

$$
\omega=\omega_{0}\left[1+\frac{3}{8} \frac{k_{3}}{k_{1}} \bar{U}^{2}\right] .
$$

Considering an harmonic driving term $F(t)=\bar{F} \cos (\omega t)$ of angular frequency $325 \omega$ close to the natural angular frequency $\omega_{0}$ defined through (7), one obtains a relation between the amplitude $\bar{F}$ and the frequency $\omega$ of the driving force and the amplitude $\bar{U}$ of the resonator's forced vibration:

$$
\left(\frac{\bar{F}}{k_{1}}\right)^{2}=\left(2\left(1-\frac{\omega}{\omega_{0}}\right) \bar{U}+\frac{3}{4} \frac{k_{3}}{k_{1}} \bar{U}^{3}\right)^{2}+\left(\frac{b}{m \omega_{0}} \bar{U}\right)^{2}
$$

from which it is possible to compute the frequency response of the resonator:

$$
\omega=\omega_{0}+\frac{3}{8} \frac{k_{3}}{\sqrt{k_{1} m}} \bar{U}^{2} \pm \sqrt{\left(\frac{\bar{F}}{2 \sqrt{k_{1} m} A}\right)^{2}-\left(\frac{b}{2 m}\right)^{2}} .
$$

Note that, the method of successive approximations is only one of the possisystems. In [110], the harmonic balance method is proposed as a valid alternative, while in [111], the energy balance method is employed for the solution of a non-linear dynamic system. Younis et al. [112] proved that perturbation methods [113] such as the method of multiple scales [114] provide better and 335 
non-linear regime. Finally, another possibility for the solution of non-linear dynamic equations, is the use of numerical techniques such as the continuation method with arc length control [115, 87, 21].

\subsection{Parametric resonance}

Another possible example of peculiar dynamic behavior in MEMS is the parametric resonance described by the Mathieu equation. It is a linear differential equation with variable (periodic) coefficients; by considering $U$ as the one degree of freedom of the system, this equation reads

$$
\ddot{U}+\frac{\omega}{Q} \dot{U}+\left[1+\frac{\Delta k}{k} \cos (2 \omega t)\right] \omega^{2} U=\frac{F}{m} \cos (\omega t+\phi)
$$

where $\lambda \equiv \frac{\Delta k}{k}$ is the fractional stiffness change, $F$ the amplitude of the timedependent forcing term, $Q$ the quality factor and $\omega$ the natural frequency. When $\lambda=0$, the device is a linear resonator and the sensitivity to force at the resonance frequency $\omega$ is $Q / k$. When $\lambda \neq 0$, the device is a parametric resonator and the excess parametric gain depends on the phase $\phi$ of the $1 \omega$ signal $F$ relative to the $2 \omega$ pump. The total force-to-displacement gain at resonance is $G(\phi) Q / k$, where the phase-dependent parametric gain is given by (see [116]):

$$
G(\phi)=\left[\left(\frac{\cos (\phi)}{1+\frac{Q \Delta k}{2 k}}\right)^{2}+\left(\frac{\sin (\phi)}{1-\frac{Q \Delta k}{2 k}}\right)^{2}\right]^{1 / 2} .
$$

Maximum amplification occurs when the $1 \omega$ and $2 \omega$ signals are phase shifted by $\phi= \pm 90^{\circ}$, and the system becomes self-oscillating when the stiffness change reaches a critical threshold, $\lambda_{c}=2 / Q$, a condition known as autoparametric oscillation.

It is common to have parametrically amplified MEMS devices that are also subjected to other sources of nonlinearities (e.g. electrostatic actuation or high quality factor). Their dynamics is usually modelled through the non-linear Mathieu equation that reads:

$$
\ddot{U}+\frac{\omega}{Q} \dot{U}+\left[1+\frac{\Delta k}{k} \cos (2 \omega t)+\frac{k_{3}}{k} U^{2}\right] \omega^{2} U=\frac{F}{m} \cos (\omega t+\phi) .
$$


where $k_{3}$ is the cubic term that is added to eq. (11) to take into account the difelectrostatic driving and sensing of each resonator is obtained by means of two 


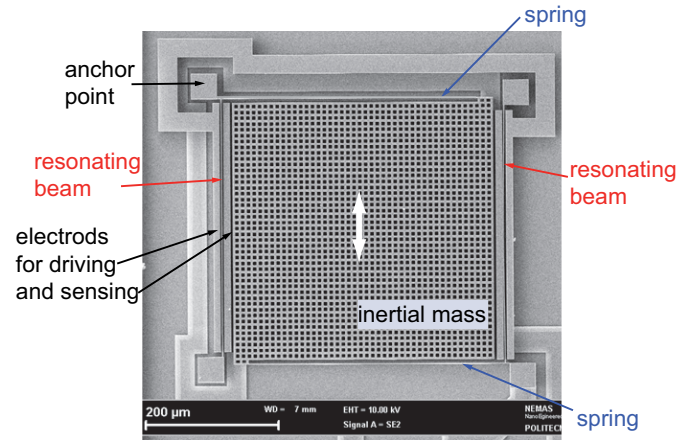

(a)

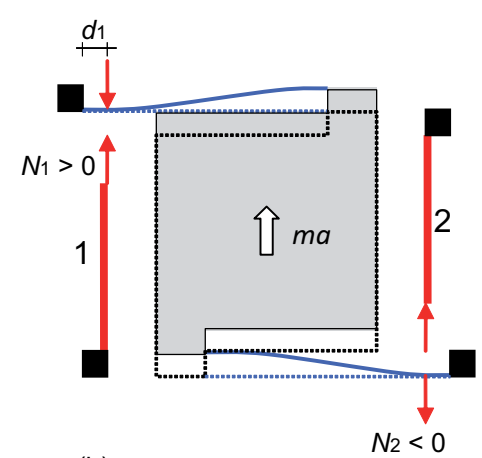

(b)

Figure 2: (a) SEM image of the resonant accelerometer. (b) Effect of external acceleration $a$. Rielaborated from: [123], Fig. 1 and 6. Reproduced with permission of IEEE.

parallel electrodes attached to the substrate.

An external acceleration $a$ makes the inertial mass $m$ translate thus inducing tension and compression of the same magnitude in the two resonators (Fig. 2b). The axial forces produce a change of the mechanical stiffness and hence of the resonance frequency of the two oscillators which provides a differential sensing of the external acceleration.

The dynamic response of the two L-shaped resonators is computed through eq. (5). The resonators are modelled as slender beams, of cross section $A$, inertia moment $J$ and Young's modulus $E$, axially constrained at both ends, subject to axial forces $P_{0}$ induced by external acceleration, oscillating in bending due to electrostatic actuation. The L-shaped configuration relaxes the axial constraint at one end and this is modelled through an equivalent axial spring $k_{a}$, see Fig. $400 \quad 3$.

These resonators, under large displacement oscillations, exhibit non-linear behavior due to the parallel plate actuation scheme (electrostatic non-linearity) and to the axial stretching (mechanical non-linearity). As demostrated in [126], by expressing the transversal displacement as the product of the eigenfunction $\Psi(x)$ (describing the beam deformation) and the transversal displacement of the mean point of the beam $U(t)$ and using Hamilton's principle, one obtains the 


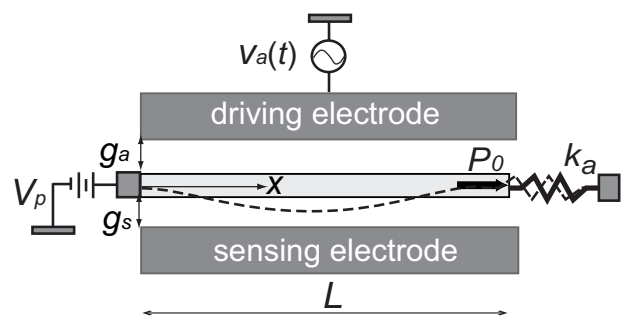

Figure 3: Schematic view of the L-shaped resonator with the actuation scheme.

1-dof equation (5) with $p=3$ and the equivalent mass, stiffnesses and damping coefficient expressed as:

$$
\begin{gathered}
m=\int_{0}^{L} \rho A \Psi^{2} \mathrm{~d} x \\
k_{1}=k_{m 1}+k_{G}+k_{e 1} \\
k_{m 1}=\int_{0}^{L} E J\left(\Psi^{\prime \prime}\right)^{2} \mathrm{~d} x \\
k_{G}=P_{0} \int_{0}^{L}\left(\Psi^{\prime}\right)^{2} \mathrm{~d} x \\
k_{e 1}=-\epsilon_{0} w V_{p}^{2}\left(\frac{1}{g_{a}^{3}}+\frac{1}{g_{s}^{3}}\right) \int_{0}^{L} \Psi^{2} \mathrm{~d} x \\
k_{2}=k_{e 2}=-\frac{3}{2} \epsilon_{0} w V_{p}^{2}\left(\frac{1}{g_{a}^{4}}-\frac{1}{g_{s}^{4}}\right) \int_{0}^{L} \Psi^{3} \mathrm{~d} x \\
k_{3}=k_{m 3}+k_{e 3}=\frac{1}{2} \frac{k_{a} E A}{k_{a} L+E A}\left[\int_{0}^{L}\left(\Psi^{\prime}\right)^{2} \mathrm{~d} x\right]^{2}-2 \epsilon_{0} w V_{p}^{2}\left(\frac{1}{g_{a}^{5}}+\frac{1}{g_{s}^{5}}\right) \int_{0}^{L} \Psi^{4} \mathrm{~d} x(20) \\
b=\int_{0}^{L} b^{*} \Psi^{2} \mathrm{~d} x
\end{gathered}
$$

where $w$ is the out-of-plane thickness of the electrodes and $b^{*}$ is the viscous coefficient. For ideal structures, with symmetric gaps $g_{a}=g_{s}$, the non-linear second order term disappears (eq. 19) thus recovering the case of a Duffing oscillator. The linear mechanical stiffness accounts for the flexural stiffness of the beam $k_{m 1}$ and for the geometrical stiffness $k_{G}$ which depends on the axial force. It is this latter term that allows for the frequency sensing of the external acceleration in the resonant accelerometer. The effect of the first-order electrical stiffness is a downward shift of the resonator natural frequency. The third order stiffness (eq. 20) has two contributions. The first one is the mechanical 

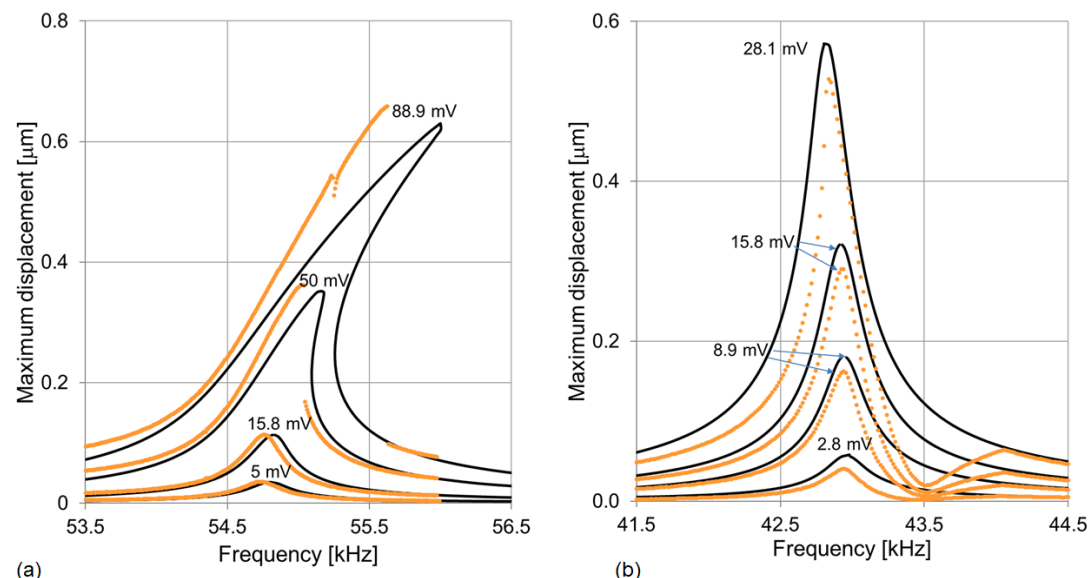

Figure 4: Spectral response of the L-shaped resonator for different actuation voltages: comparison experiments (orange points) - theoretical prediction (black lines). (a) $V_{p}=4 V$; (b) $V_{p}=9 \mathrm{~V}$.

hardening term which accounts for the membrane effect: it disappears if $k_{a} \rightarrow 0$, i.e. if there is no axial constraint and it is maximum for the doubly clamped beam, without the transversal short arm $\left(k_{a} \rightarrow \infty\right)$. The second term is due to the electrostatic actuation, it grows with the bias potential $V_{p}$ and it is negative, thus it can mitigate the hardening effect generally associated with the third-order term of the elastic stiffness. By properly choosing the biasing voltage one can, in principle, compensate the mechanical nonlinearities with the electrical ones in order to obtain a linear behavior for a wider range of applied forces [127], i.e., for a wider range of actuation voltages as shown in Fig. 4a-b.

\section{Hardening behavior under varying temperature conditions in a Double Ended Tuning Fork for a resonant accelerometer}

The present Section discusses the effects of the temperature variation in the non-linear dynamic response of the Double Ended Tuning Fork resonator $(\mathrm{DETF})$ contained in the resonant accelerometer described in $[128,129]$ and shown in Fig. 5. 

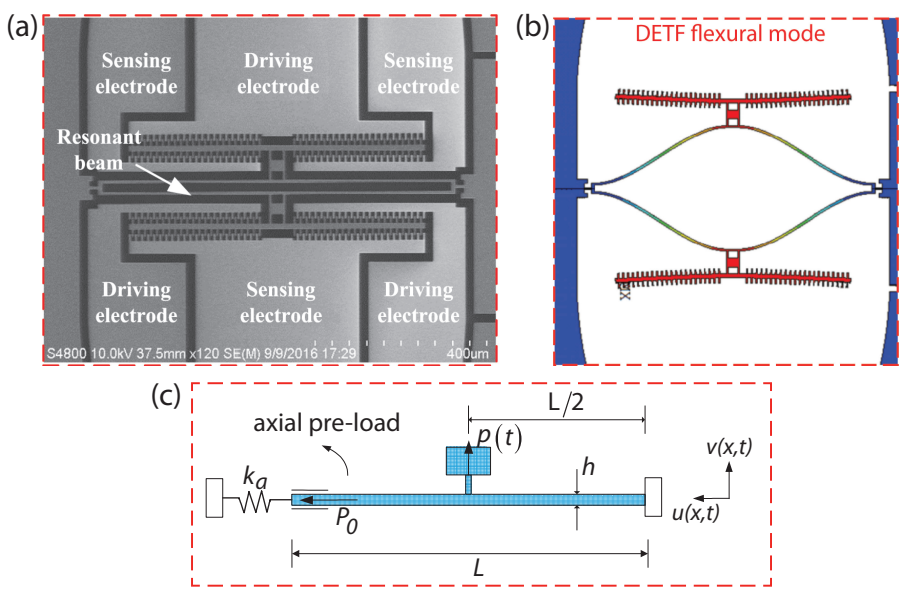

Figure 5: (a) SEM Close-up view of the DETF resonator discussed in [129]. (b) Schematic view of the DETF resonator. (c) Schematic view of a single resonant beam.

The DETF is electrostatically actuated according to its flexural mode (see Fig. 5b) through two sets of comb finger actuators (see Fig. 5a). In absence of external acceleration, the DETF oscillates at its natural frequency while the proof mass is fixed. When an external acceleration acts along the $x$-axis, the proof mass of the accelerometer, not shown in Fig. 5, translates in the same direction and the DETF experiences tensile/compressive loads (see [129] for more details), thus changing the frequency of its flexural mode. By electrostatically detecting the frequency variation of the resonator through comb fingers in a differential scheme, it is possible to measure the external acceleration acting on the sensor.

Similarly to what shown in the previous Section, the inertia force induced by the external acceleration is considered as a constant axial pre-load, $P_{0}$, in the beam while the force exherted by the driving comb fingers on the resonator is modelled through the transversal dynamic load $p(t)$. To better schematize the real behavior of the resonator, the beam is axially constrained at both ends, with an axial spring of stiffness $k_{a}$ at one end to represent the constraint given by the presence of the micro-lever mechanism (see Fig. 5c).

To take into account the effect of the temperature variation in the dynamic 
response of the resonator under study, the Young's modulus and the thermal expansion coefficient of silicon were considered temperature dependent according to [130] and [131] respectively. Let $T(t)$ be the temperature and $\Delta T$ the temperature shift with respect to $T(0)=T_{0}$; the temperature-dependent Young's modulus $E(T)$ and thermal expansion coefficient $\alpha(T)$ become:

$$
\begin{aligned}
& E(T)=E(298.16 \mathrm{~K})\left(1+T C E_{1} \cdot \Delta T+T C E_{2} \cdot \Delta T^{2}\right) \\
& \alpha(T)=-4 \times 10^{-12} T^{2}+8 \times 10^{-9} T+4.7 \times 10^{-7},
\end{aligned}
$$

with $T C E_{1}=-63.82 \mathrm{ppm} / \mathrm{K}, T C E_{2}=-51.99 \mathrm{ppb} / \mathrm{K}^{2}$ and $E(298.16 \mathrm{~K})=$ 168.9 GPa for $<110>$ monocrystalline silicon.

The equation of motion describing the non-linear dynamics of the resonator under varying temperature conditions, was obtained in [129]: it is a particular case of eq. (5) with $p=3$ and $k_{2}=0$.

Equivalent mass, stiffness and load are in this case given by:

$$
\begin{gathered}
m=\int_{0}^{L} \rho A \Psi^{2} \mathrm{~d} x+m_{c o m b}, \\
k_{1}=k_{m 1}+k_{G}, \\
k_{m 1}=\int_{0}^{L} E(T) J\left(\Psi^{\prime \prime}\right)^{2} \mathrm{~d} x, \\
k_{G}=\left(P_{0}-\frac{k_{a} E(T) A \cdot \alpha(T) \Delta T}{k_{a} L+E(T) A} L\right) \int_{0}^{L}\left(\Psi^{\prime}\right)^{2} \mathrm{~d} x, \\
k_{3}=k_{m 3}=\frac{1}{2} \frac{k_{a} E(T) A}{k_{a} L+E(T) A}\left[\int_{0}^{L}\left(\Psi^{\prime}\right)^{2} \mathrm{~d} x\right]^{2}, \\
b=\int_{0}^{L} b^{*} \Psi^{2} \mathrm{~d} x, \\
F=p(t)+\frac{2 \alpha(T) \Delta T E(T) J}{h} \int_{0}^{L} \Psi^{\prime \prime} \mathrm{d} x, \text { with } p(t)=\frac{\partial C}{\partial x} V_{P} v_{a},
\end{gathered}
$$

where $v_{a}=\left|v_{a}\right| \cos \left(\omega_{F} t\right)$ is the driving voltage, $C$ is the capacitance measured by the comb fingers $V_{p}$ is the bias voltage and $h$ is the beam height (see Fig. $5 c)$.

From the above equivalent coefficients it can be remarked that the geometrical stiffness $k_{G}$ and the equivalent load $F$ (i.e. static contribution in the forcing term) now depend on the temperature. Moreover, no electrostatic stiffness appears in this case due the use of comb finger actuators instead of parallel plate ones. 


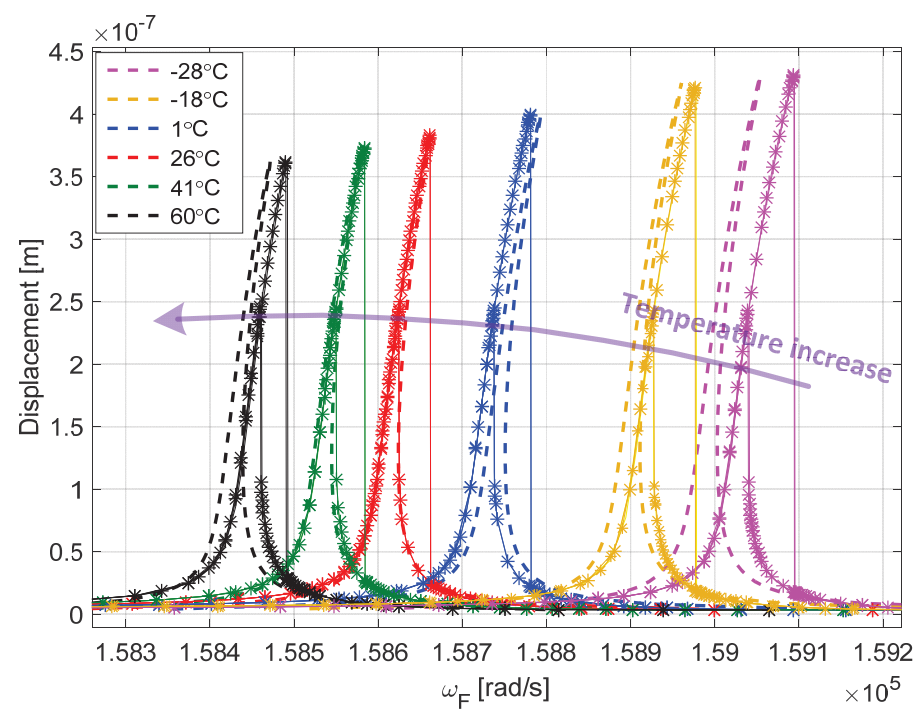

Figure 6: Frequency response of the DETF resonator at different temperatures in the range $\left[-30^{\circ} \mathrm{C} ; 60^{\circ} \mathrm{C}\right]$. A driving voltage of $10 \mathrm{mV}$ of amplitude and a bias voltage of $10 \mathrm{~V}$ is applied to the device. Source: [129], Fig. 10.

In Fig. 6, a comparison between the frequency responses of the resonator measured at different temperatures (stars in Fig. 6) and the theoretical curves computed (dashed line in Fig. 6) solving eq. (5) with eqs. (23)-(29) is shown. The natural frequency of the resonator and the maximum vibration amplitude at resonance decrease with increasing temperatures.

The results of Fig. 6 show, as expected, that temperature variations can highly influence the dynamic response of micro-resonators and consequently induce complex scenarios in the device dynamics.

\section{Softening behavior in a torsional resonator for a resonant accelerom- eter}

The present Section focusses on torsional resonators which are the key component of the out-of-plane resonant accelerometer proposed in [132] and shown in Fig. 7. In this case the proof mass is attached to the substrate through a torsional spring and, in the presence of an out-of-plane acceleration, tilts around 


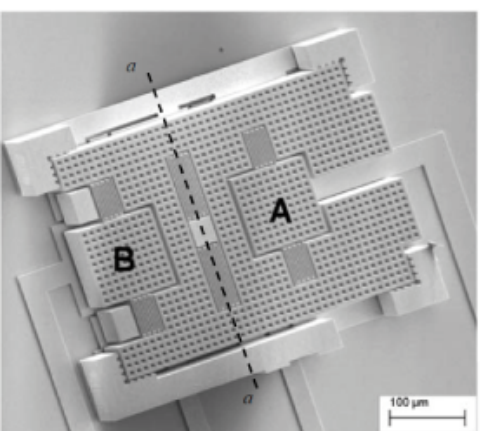

(a)

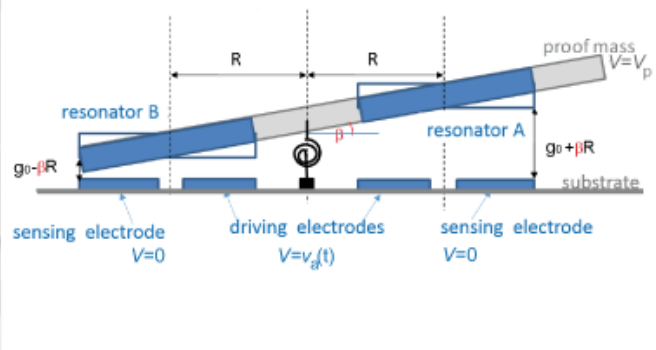

(b)

Figure 7: (a) SEM image of the out-of-plane accelerometer with two torsional resonators $\mathrm{A}$ and B; (b) schematic side view of the accelerometer inclined by an external acceleration. Source: [133], Fig. 1. Reproduced with permission of Elsevier.

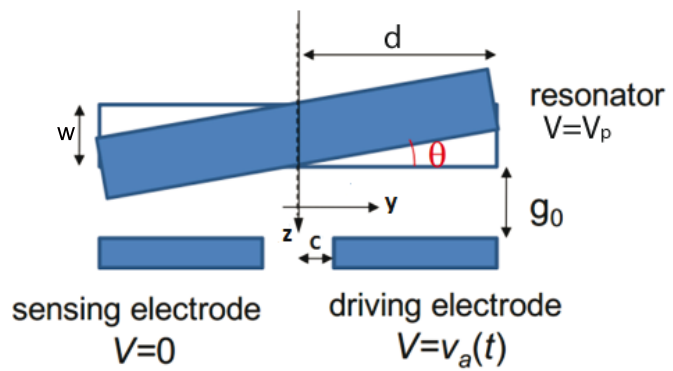

(a)

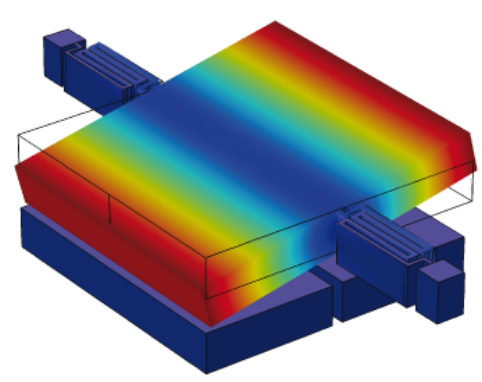

(b)

Figure 8: (a) Electrostatic actuation scheme of the torsional resonator, (b) first torsional mode of the torsional resonator. The contour plot of the displacement field is shown in color.

the axis $a-a$. Two small square masses $\mathrm{A}$ and $\mathrm{B}$, the torsional resonators, are attached to the proof mass and kept at resonance during the functioning by electrostatic actuation (Fig. 8). When an external acceleration is applied, the proof mass rotates and the gap $g_{0}$ between the resonators and the electrodes changes. Since the torsional electrostatic stiffness depends on the gap, through a relation similar to eq. (3), it changes and makes the frequency vary in opposite directions in resonators $\mathrm{A}$ and $\mathrm{B}$, thus allowing for a differential acceleration sensing. 
Each one of the two torsional resonators can be described as a one degree of freedom system (the dof is the rotation of the mass $\theta(x, t)=\Theta(t) \Psi(x)$, with $\Psi$ a proper shape function) and the equation governing its dynamics is again expressed by eq. (5) truncated at the third order with $U(t) \equiv \Theta(t)$. Note that, since in this case the degree of freedom is an angle, the equivalent mass, stiffnesses and forcing term change, accordingly, their dimensions and read

$$
\begin{array}{r}
m=2 \int_{0}^{L} \rho J_{p} \Psi^{2} \mathrm{~d} x+\rho J_{m a s s}, \\
k_{1}=k_{m}+k_{e 1}, \\
k_{m}=2 \int_{0}^{L} G J_{t}\left(\Psi^{\prime}\right)^{2} \mathrm{~d} x, \\
k_{e 1}=-\frac{2 \epsilon_{0} B}{g_{0}^{3}} V_{p}^{2}\left(\frac{d^{3}}{3}-\frac{c^{3}}{3}\right), \\
k_{3}=k_{e 3}=-\frac{4 \epsilon_{0} B}{g_{0}^{5}} V_{p}^{2}\left(\frac{d^{5}}{5}-\frac{c^{5}}{5}\right), \\
F(t)=\frac{\epsilon_{0} B}{g_{0}^{2}} V_{p} v_{a}(t)\left(\frac{d^{2}}{2}-\frac{c^{2}}{2}\right), \\
b=\int_{0}^{L} b^{*} \Psi^{2} \mathrm{~d} x,
\end{array}
$$

where $d$ and $c$ are defined in Fig. 8, $J_{t}$ is the torsional moment of inertia, $G$ the shear elastic modulus, $J_{p}$ is the polar moment of inertia of a single spring of

length $L, \rho J_{\text {mass }}$ is the centroidal mass moment of inertia of the rigid mass and $F(t)$ is now the external torque applied to sustain the oscillation. Note that the term $k_{e 3}$, coming from the Taylor expansion of the electrostatic moment arrested at the third order [133] is negative and this implies a softening effect in the frequency response of the torsional resonator as usually happens when only electrostatic parallel plates nonlinearities are taken into account in the model. The second order term $k_{e 2}$ is zero for symmetry reasons.

In Fig. 9a the frequency-response curve for a torsional resonator (the geometric dimensions used are the ones of the torsional resonator of the $z$-axis resonant accelerometer studied in [134]) is shown for different values of the actuation voltage $\left|v_{a}\right|$ at the fixed polarization voltage $V_{p}=6 \mathrm{~V}$.

For significantly high $\left|v_{a}\right|$, there is a range of $\omega$ where $\Theta$ becomes a multival- 
ued function and for a given $\omega$ three solutions are available (see Fig. 9a, points $P_{1}, P_{2}$ and $\left.P_{3}\right)$. The critical value of $\left|v_{a}\right|$ after which the response becomes a multi-valued function of the frequency, is:

$$
\left|v_{a_{c}}\right|=\sqrt{\frac{8 b^{3} \omega_{0}^{3}}{3\left|k_{3}\right|}} \frac{2 g_{0}^{2}}{\epsilon_{0} L V_{p}\left(d^{2}-c^{2}\right)} .
$$

Note that for $\left|v_{a}\right| \ll\left|v_{a_{c}}\right|$ the results are linear: the frequency response curves are symmetric and represent the solution in a very narrow band around the resonant frequency.

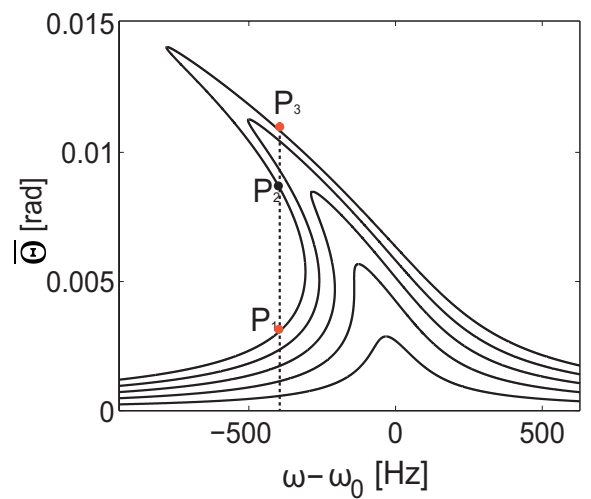

(a)

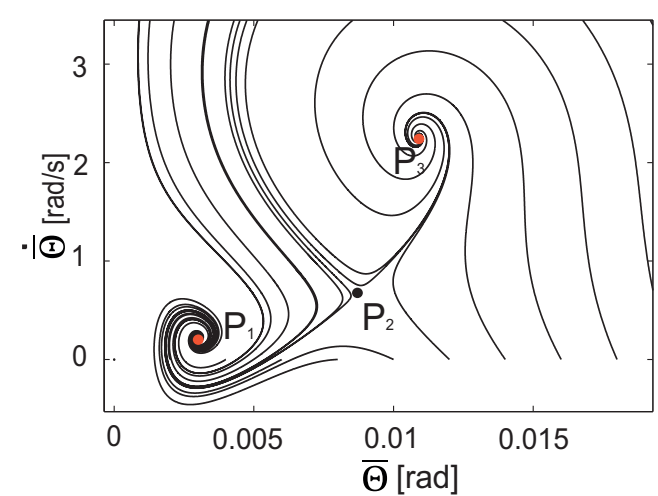

(b)

Figure 9: Dynamic response of the torsional resonator. a) Frequency-response curve with $V_{p}=6 \mathrm{~V}$ and $\left|v_{a}\right|=10,20,30,40,50 \mathrm{mV}$; b) trajectories of the system in the state plane: the two stable steady state solutions $\left(P_{1}, P_{3}\right)$ and the unstable one $\left(P_{2}\right)$ are marked with the red and black dots respectively, [135].

When $\left|v_{a}\right|>\left|v_{a_{c}}\right|$, the frequency response is non-linear and the effect of the nonlinearity is to bend the amplitude curve and distort the phase curve: in both cases multivalued regions are formed and, consequently, jump phenomena arise (see $[136,137,138])$.

The third order approximation of the electrostatic moment gives good results for moderate values of $\left|v_{a}\right|$. To see the limit of this approximation, one should consider further terms. When terms up to the fifth order are considered, eq. (5) with:

$$
k_{5}=k_{e 5}=-\frac{6 \epsilon_{0} B}{g_{0}^{7}} V_{p}^{2}\left(\frac{d^{7}}{7}-\frac{c^{7}}{7}\right)
$$


and the other coefficients defined in (30)-(36), solved through the method of successive approximations, gives:

$$
\omega=\omega_{0}+\frac{3}{8} \frac{k_{3}}{\sqrt{k_{1} m}} \bar{\Theta}^{2}+\frac{5}{16} \frac{k_{5}}{\sqrt{k_{1} m}} \bar{\Theta}^{4} \pm \sqrt{\left(\frac{\bar{F}}{2 \sqrt{k_{1} m} \bar{\Theta}}\right)^{2}-\left(\frac{b}{2 m}\right)^{2}}
$$

where $\bar{\Theta}$ is the maximum rotation angle and $\bar{F}$ is the amplitude of the forcing electrostatic moment.

Figure 10, taken from [139], shows the comparison between the analitycal prediction obtained with the different approximations, the numerical results obtained by time integration (without Taylor expansions approximation) and the experimental results on a torsional resonator fabricated through the Thelma ${ }^{\circledR}$ surface micromachining process by ST-Microelectronics. From Fig. 10 it is evident that it is not possible to describe the real behavior of the torsional resonator when the amplitude of rotation increases and the high non-linear regime is entered, with the analytical approximation (39). The inclusion of other terms in the Taylor approximation would not improve results. Further analytical methods (e.g. multiple scale or harmonic balance) must be then considered as mentioned in Section 3.1 to understand the source of this discrepancy. On the other hand, the numerical solution well reproduces the experimental response of the resonator thus proving the effectiveness of the proposed one degree of freedom model.

Figure 11 shows the limit pull-in domain in the $V_{p}-\left|v_{a}\right|$ plane. Experimental frequency responses obtained for $V_{p}-\left|v_{a}\right|$ combinations above the upper bound (orange points in Fig. 11) show dynamic pull-in (asymptotic trend for large rotation amplitude) while for $V_{p}-\left|v_{a}\right|$ combinations under the lower bound (black point in Fig. 11), the classic linear or hysteretic behavior is obtained. The numerical lower bound, consequently, allows to define the safe operation region of the device that is, usually, of fundamental importance during the design process. 


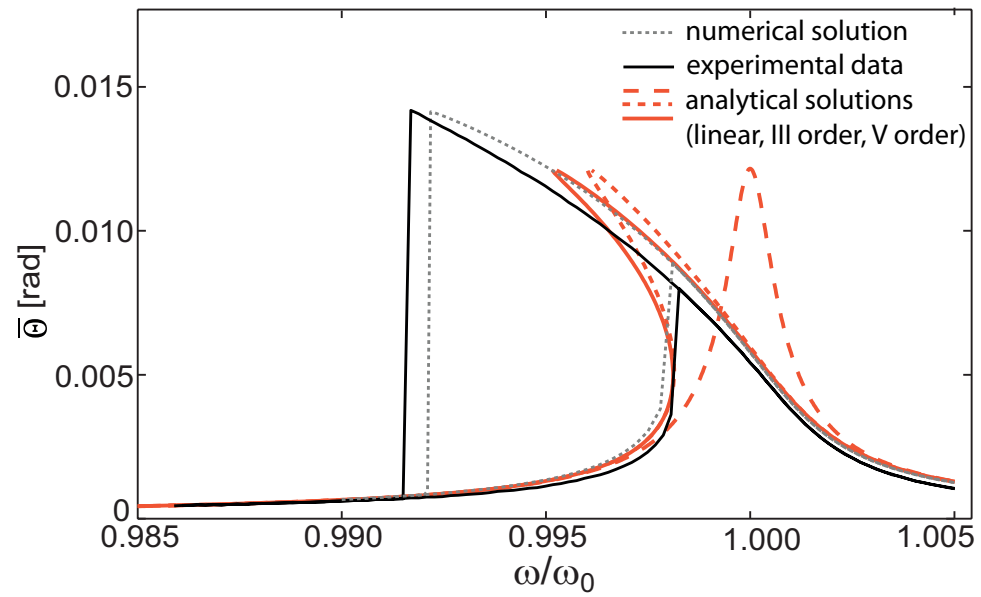

Figure 10: Frequency response of the torsional resonator for $V_{p}=5 \mathrm{~V}$ and $\left|v_{a}\right|=100 \mathrm{mV}$ : linear, III and V order analytical approximations (gray curves), numerical prediction (dotted dark gray) and experimental curves (continuous black); both forward and backward frequency sweeps are shown, [139].

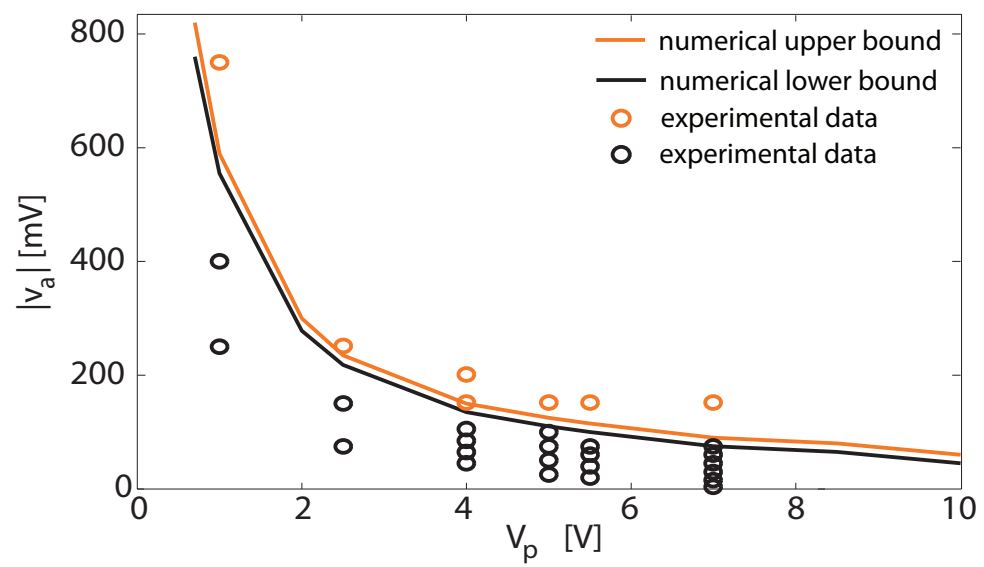

Figure 11: Limit pull-in domain in the bias voltage-actuation voltage plane: numerical prediction (lines) and experimental measurements (dots). The couples of bias and actuation voltages leading to dynamic pull-in are shown in orange, while black corresponds to values of voltages leading to a dynamic response immune to pull-in, [139]. 
7. Geometric nonlinearities in MEMS elastic springs for beating heart Coriolis-based gyroscopes

MEMS gyroscopes are devices that measure the angular velocity. Their

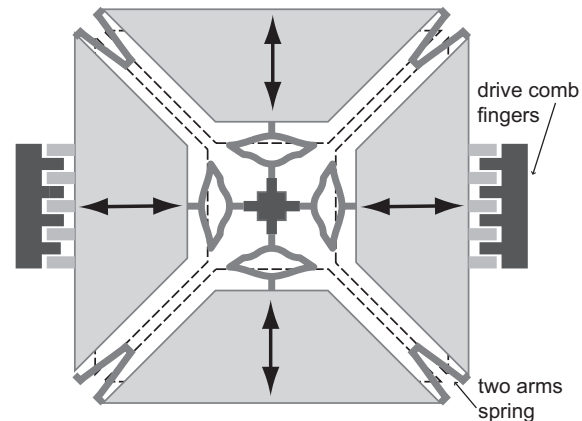

(a)

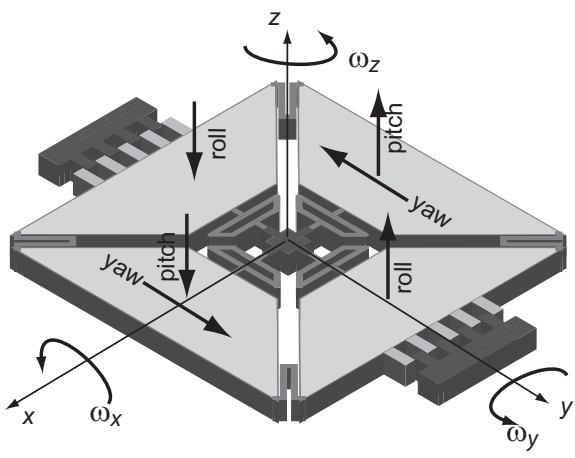

(b)

Figure 12: Scheme of the 3-axis beating-heart gyroscope: (a) in-plane drive mode, (b) scheme of sensing modes for roll, pitch and yaw as a consequence of Coriolis' forces.

The mechanical design of CVG is complex as it implies the presence of actuators that sustain the drive mode of oscillating parts and of several deformable 


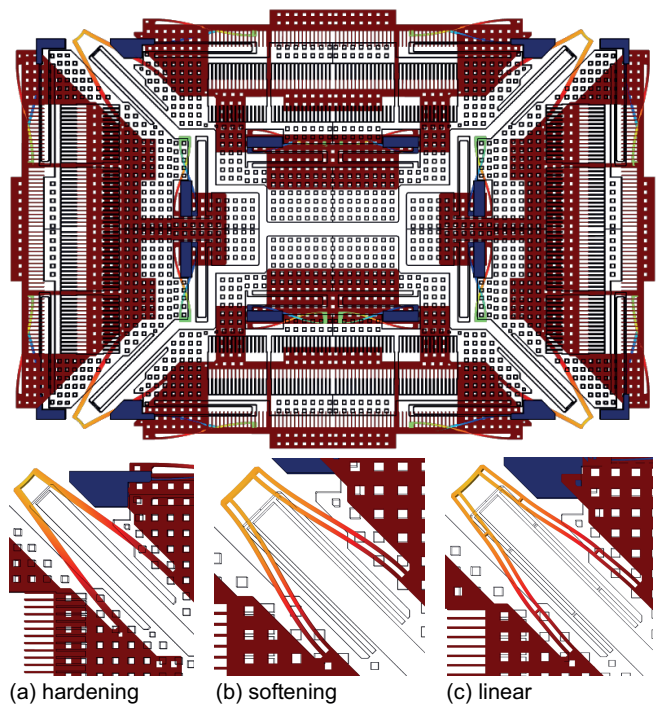

Figure 13: Eigen shape-function of the first mode of the quad-mass test structure with (a) hardening, (b) softening and (c) liner coupling springs. The contour of the displacement field is shown in color.

portions which allow the movement of the various masses composing the device.

In the present Section the focus is on the special deformable elements, twoarms springs, which connect the moving masses transferring the horizontal motion of one mass to the vertical motion of the other mass (Fig. 13) and which can enter in softening or hardening mechanical regimes [141].

It is possible to schematize half of the elastic coupling spring by putting a slider on one end inclined by $45^{\circ}$ to represent the connection between one arm of the coupling spring and the proof mass and a symmetry condition on the other end (see Fig. 14a).

Three different cross sections of the coupling springs have been considered (see Figs. 13a-c) to prove the tunability of the non-linear response of such elastic elements. While for a beam with a compact cross section the non-linear effect leads always to a hardening response [141], a softening behavior can be obtained by considering built-up beams. In particular, reference is made to a battened

beam as illustrated in Fig. 14. More examples of battened beams can be found 
(a)

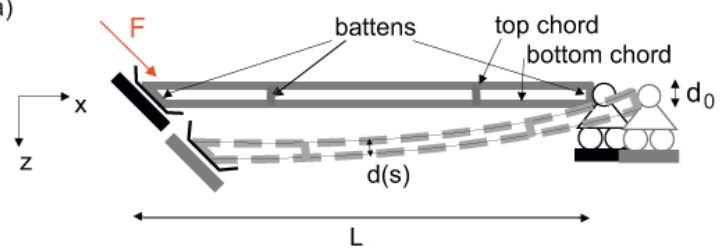

(b)

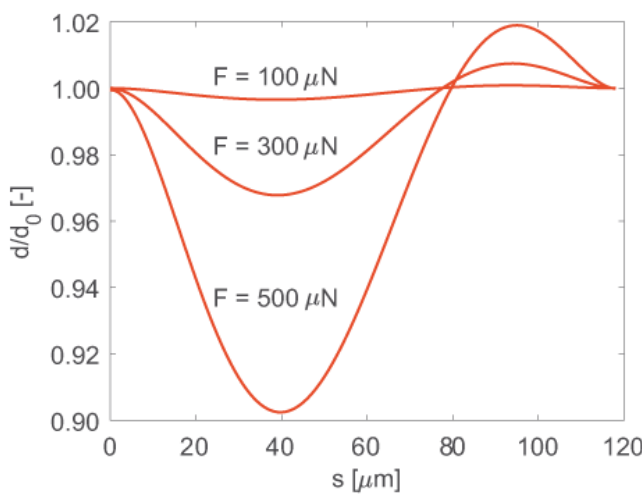

Figure 14: (a) Schematic view of a built-up beam with 4 battens in the undeformed and deformed configurations. (b) Variation of the normalized distance between the two chords for different values of the applied force $F$ in a 2-battens beam.

in $[142,143]$.

The softening response of such beams can be explained by considering that, when the battened beam deflects, the two chords are subject to axial forces of opposite sign which, in the large displacement range, influence the elastic curve of the chords between the successive battens and hence cause a change of the relative distance $d(s)$ as shown in Fig. 14b. In particular, for the deflection sketched in Fig. 14a, the lower chord is stretched and hence its transversal displacement is lower than the one of the upper compressed chord. Therefore the distance $d$ varies along the beam $d=d(s)$, decreases in the central part and the equivalent inertial moment decreases as well leading to a progressive reduction of the bending stiffness $E J_{e q}$. This softening effect can compensate or even overcome the hardening one.

An analytical model able to describe the non-linear mechanical stiffness of a coupling spring made by beams of compact section as well as battened beams is 

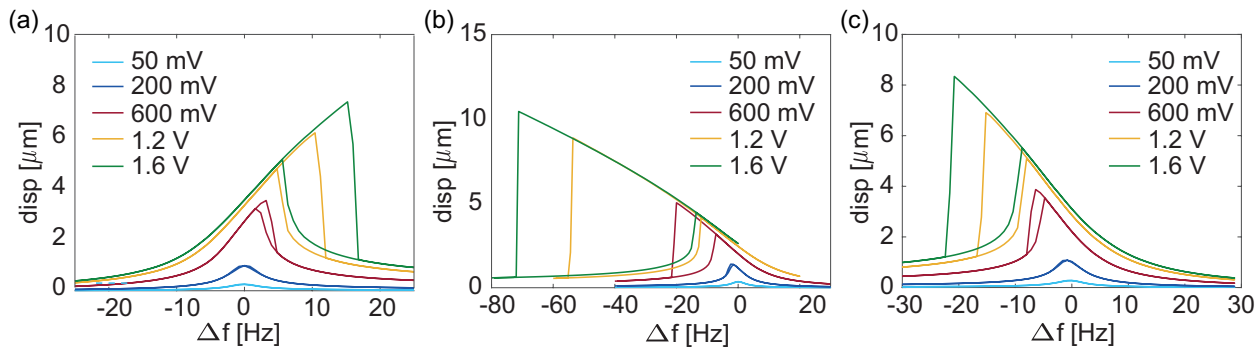

Figure 15: Experimental dynamic responses of the (a) compact, (b) two battens and (c) four battens.

reported in [141]. By moving from the compact to the built-up section one can pass from a hardening behavior to a softening one; intermediate linear responses can be obtained by changing the number of battens.

The experimental frequency responses of the three test structures (Fig. 15) show the hardening behavior of the spring with compact section and the softening behavior of the two-chords springs. The presence of multiple battens lower the softening effect of the coupling spring designed with only two battens at the ends: for displacement amplitudes similar to the ones shown in Fig. 15b, the frequency shift induced by nonlinearities is in fact reduced by a factor of 4 .

\section{Parametric resonance in a Disk Ring Gyroscope}

Disk or ring gyroscopes operate using two orthogonal flexural radial vibration modes. These mode shapes have deformation proportional to $\cos (n \vartheta)$ and $\sin (n \vartheta)$, where $n$ is the mode number. Generally the $2 \vartheta$ or $3 \vartheta$ modes, which are separated from each other by $45^{\circ}$ and $30^{\circ}$ respectively, are used for gyroscope operation.

The disk resonating gyroscopes have a symmetric structure with central anchor and concentric internal rings; an example is shown in Fig. 16 taken from [144]. The device is realized by a single-crystal silicon slotted disk supported by a central cylindrical anchor and surrounded by capacitive electrodes used to force and sense vibration; it has $2 \vartheta$ orthogonal elliptical modes, separated by $45^{\circ}$, see Fig. 16b. 
a)
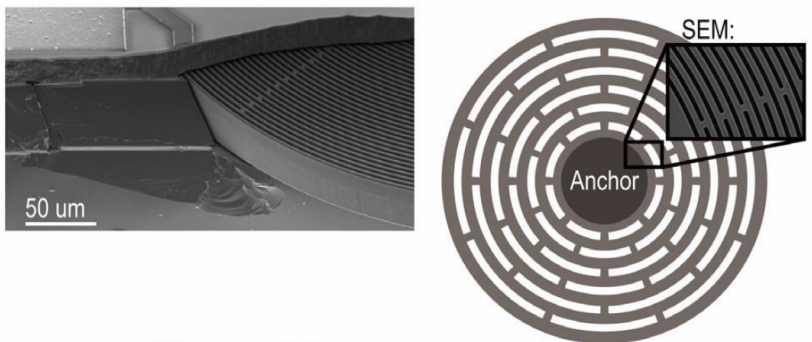

b)

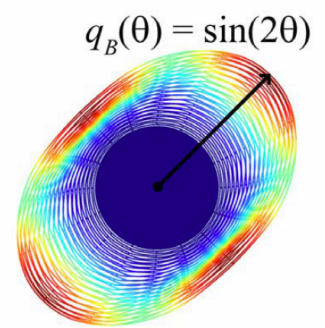

$45^{\circ}$ Mode

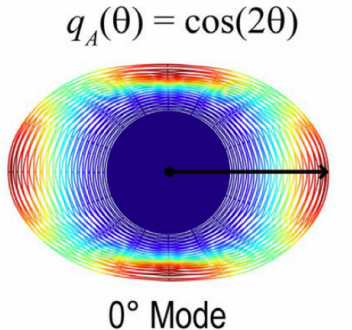

Figure 16: Disk Resonator Gyroscope (DRG) fabricated in $<100>$ silicon in Stanford's Episeal process proposed by researches at the Robert Bosch Research and Tecnology Center in Palo Alto and then demonstrated in a close collaboration with Stanford University. (a) SEM of DRG and drawing showing DRG shape, with inset SEM of rings. (b) Orthogonal elliptical mode shapes, with contours of the displacement. Source: [144], Fig. 1. Licensed under a Creative Commons Attribution 4.0 International License http://creativecommons.org/licenses/by/4.0/.

The DRG shown in Fig. 16a is here employed to show the phenomenon of the parametric resonance in MEMS devices. When the large displacement regime is entered, non-linear mechanical coupling between the two degenerate modes of the DRG (Fig. 16b) leads to self-induced parametric amplification of the Coriolis force input. The self-induced parametric amplification has been experimentally demonstrated for the first time in [144] while several subsequent works aim at providing a complete modelling of the phenomenon (e.g. [145, 146, 147]).

Figure 17a shows a lumped element model for the device: the stiffness of the sensing mode is modulated in time by the non-linear elastic effects induced by the mechanical coupling of the two modes. In particular, the $\Delta k$, which is the variation in stiffness of the sense axis, depends on the drive axis displacement as shown in Fig. 17. Since the mode shape is two-fold symmetric, the stiffness 
(a)

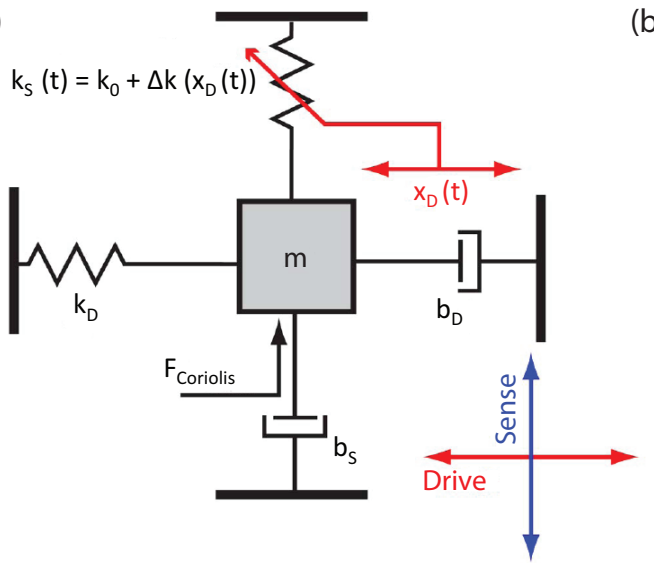

(b)
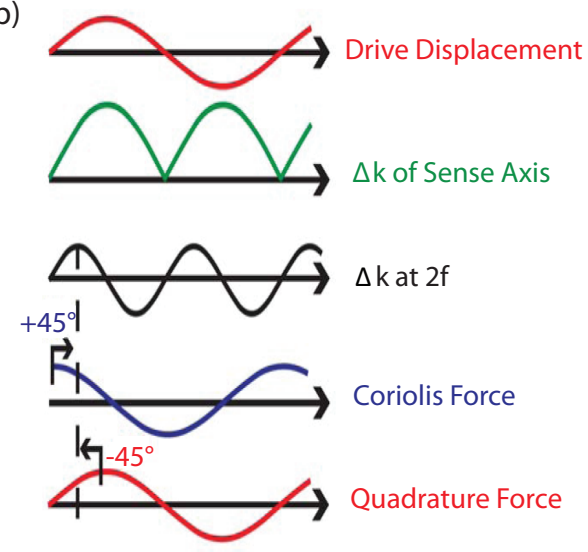

Figure 17: Lumped element model of gyroscope: the displacement of the drive axis modulates the stiffness of the sense axis at twice the resonant frequency, thus parametrically amplifying Coriolis force and electrostatic inputs to the sense axis. Source: [144], Fig. 3. Licensed under a Creative Commons Attribution 4.0 International License http://creativecommons.org/licenses/by/4.0/.

change is not sensitive to the sign of the displacement and $\Delta k$ approximates a rectified sine wave. The $2 \omega$ component of this rectified sine wave has a $\phi=$ $45^{\circ}$ phase shift relative to the drive axis displacement (Fig. 17b), establishing the phase relationship between the $2 \omega$ pump and $1 \omega$ signal waveforms (see eq. (11)).

Self-induced parametric amplification was first observed by measuring the gyroscope's sensitivity to rotation rate $S_{\Omega}$ as a function of the amplitude of the driven mode $\bar{x}_{D}$. When the driven mode's amplitude is small $\bar{x}_{D}<2.5 \%$, the frequency response exhibits the expected Lorentzian shape with $\Delta \omega / 2 \pi=3 \mathrm{~Hz}$. As $\bar{x}_{D}$ is increased, the scale-factor increases at a rate much greater than $\bar{x}_{D}$; an 8 -fold increase in $\bar{x}_{D}$ results in a 67 -fold increase in $S_{\Omega}$ and a two-fold reduction in $\Delta \omega$ (Fig. 18a).

${ }_{645}$ Due to the degeneracy of the two modes, which results in coupling between the two modes, the frequency shift of one mode induced by the motion of the other cannot be probed by common techniques, such as those employed by $[148,149,150]$ : the force sensitivity was probed by applying an additional elec- 

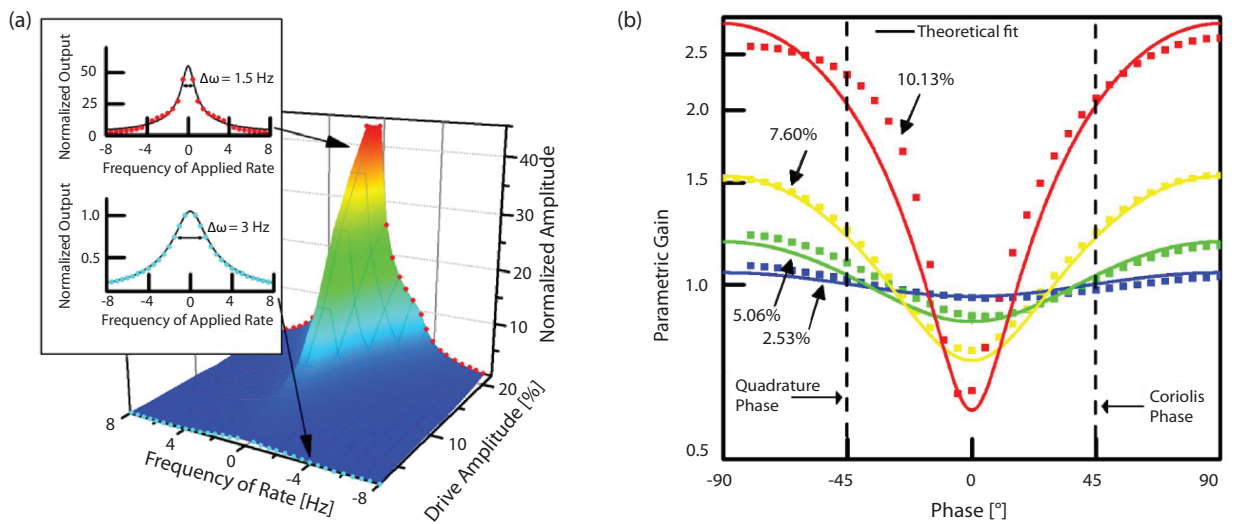

Figure 18: (a) Experimental response of the DRG to external rate. Inset shows the measured frequency response at small and large amplitudes of the driving displacement, indicating the reduced bandwidth observed at large amplitude due to the artificial increase in $Q$. (b) Parametric gain measured at different vibration amplitudes, versus phase shift between the sense axis excitation force and the drive mode's vibration. Theoretical fits are superposed in continuous lines. Source: [144], Fig. 4 and Fig. 5a. Licensed under a Creative Commons Attribution 4.0 International License http://creativecommons.org/licenses/by/4.0/.

trostatic force directly to the sensing mode with a controlled phase, $\phi$, relative to the excitation applied to the driven axis, and measuring the amplitude of the movement caused by this additional force.

The baseline motion of the sensing mode due to modal coupling, electrode misalignment and electrical feedthrough of the drive signal were subtracted, so that the resulting amplification of the force applied to the sense mode could be accurately measured.

The resulting amplification of this additional force is shown in Fig. 18b, exhibiting phase dependence consistent with parametric amplification (eq. (12)), with maximum amplification occurring at $\phi= \pm 90^{\circ}$ and minimum amplification at $\phi=0^{\circ}$.

\section{Closing remarks}

The present paper is intended to give an overview of possible non-linear mechanical responses in microsystems, with particular reference to the dynamic 
response of electrostatically actuated mechanical oscillators, important components of inertial MEMS. The modelling of non-linear behaviors in MEMS is gaining increasing attention from the scientific community due to the progressive miniaturization and the constant research of better performances of MEMS devices. Moreover, mastering of non-linear mechanical phenomena can open new perspectives if combined with readout circuits able to govern it.

After reviewing many possible sources of nonlinearities in MEMS, various practical examples of mechanical oscillators which enter non-linear dynamic regimes due to hardening and/or softening effects have been discussed. Particular attention has been focused on geometric and electrostatic nonlinearities and on the effect of the temperature on the dynamic response of MEMS oscillators. Parametric resonance has been discussed with reference to a Disk Ring gyroscope.

The examples discussed in this paper have been taken from the Authors' experience and all refer to real devices fabricated with state of the art MEMS technology.

Many other devices in which complex multi-physics behaviors arise would deserve attention. Among these, it is interesting to mention the emerging field of piezoelectric MEMS in which electro-mechanical coupling is exerted at the level of the material, thanks to the piezoelectric properties. Devices like piezo micro ultrasound transducers (PMUT) are now under study and in some cases already in the market for specific applications like range finders. In the case of PMUT, peculiar nonlinear dynamic regimes can show up when the radius over thickness ratio is very high and the vibrating membranes enter in nonlinear regime due to membrane-bending coupling and due to the influence of residual stresses and pre-deformed configurations. Micro-pumps which could find interesting applications in micro-cooling and in controlled drug delivery, show mechanical responses linked to the interaction of the movable part of the pump with the fluid with possible non-linear effects. Another example are micromirrors with electrostatic, electro-magnetic or piezoelectric actuation, used for image recognition or micro-video projection. In these devices, the complexity 
of the actuation system, coupled with large rotation angles and non-linear fluid

damping phenomena create a variety of non-linear dynamic responses.

The world of microsystems is rapidly evolving; products like inertial or pressure sensors are now considered as classic in the MEMS world and are reaching high performances, new products like PMUT, micro-pumps, micro-mirrors, resonators are ready for widespread applications. Research activities show an increasing interest for devices in which sensing is combined with pre-treatment of acquired signals also with the use of machine learning approaches. The development of high performance sensors and innovative devices with non simple actuation systems will ask for improvement in the mastering of complex nonlinear and multi-physics phenomena and probably will allow for the discovery of new phenomena coming from multiphysics interactions which have not been completely explored.

Acknowledgments - Collaborations with other groups of Politecnico di Milano and with ST-Microelectronics are gratefully acknowledged for the examples discussed in Sections 4, 6, 7. Collaboration with Nanjing University of Science and

Technology is gratefuly acknowledged for the example of Section 5. Collaborations with University of California, Davis and Stanford University are gratefully acknowledged for the example of Section 8.

\section{References}

[1] A. Corigliano, R. Ardito, C. Comi, A. Frangi, A. Ghisi, S. Mariani, Mechanics of Microsystems, Wiley, 2018.

[2] M. I. Younis, MEMS Linear and Nonlinear Statics and Dynamics., Springer, 2011.

[3] C. Acar, A. Shkel, MEMS vibratory gyroscopes. Structural approaches to improve robustness, Springer, 2009.

720

[4] S. D. Senturia, Microsystem Design, Springer US, 2001. 
[5] W. K. Schomburg, Introduction to Microsystem Design, Springer-Verlag, Berlin Heidelberg, 2015.

[6] J. J. Allen, Micro Electro Mechanical System Design, CRC Press, 2005.

[7] M. J. Madou, Fundamentals of microfabrication: the science of miniaturization, 2nd Edition, CRC Press, 2002.

[8] W. Menz, J. Mohr, O. Paul, Microsystem Technology, Wiley, 2008.

[9] M. Varanis, A. Silva, A. Mereles, R. Pederiva, Mems accelerometers for mechanical vibrations analysis: a comprehensive review with applications, J. of the Brazilian Soc. of Mech. Sciences and Eng. 40 (11) (2018) 527.

[10] X. Zou, A. A. Seshia, A high-resolution resonant mems accelerometer, in: 2015 Transducers - 2015 18th International Conference on Solid-State Sensors, Actuators and Microsystems (TRANSDUCERS), 2015, pp. 12471250.

[11] S. Wang, X. Wei, Y. Zhao, Z. Jiang, Y. Shen, A mems resonant accelerometer for low-frequency vibration detection, Sensors and Actuators A: Physical 283 (2018) $151-158$.

[12] E. Tatar, T. Mukherjee, G. K. Fedder, Stress effects and compensation of bias drift in a mems vibratory-rate gyroscope, J. of Microelectromech. Syst. 26 (3) (2017) 569-579.

[13] M. Bagherinia, M. Bruggi, A. Corigliano, S. Mariani, D. A. Horsley, M. Li, E. Lasalandra, An efficient earth magnetic field mems sensor: Modeling, experimental results, and optimization, J. of Microelectromech. Syst. 24 (4) (2015) 887-895.

[14] G. Langfelder, C. Buffa, A. Frangi, A. Tocchio, E. Lasalandra, A. Longoni, Z-axis magnetometers for mems inertial measurement units using an industrial process, IEEE Trans. Ind. Electronics 60 (9) (2013) 3983-3990. 
[15] S. Liu, H. Liang, B. Xiong, An out-of-plane electromagnetic induction based resonant mems magnetometer, Sensors and Actuators A: Physical 285 (2019) $248-257$.

[16] Y. Seo, D. Kim, N. A. Hall, Piezoelectric pressure sensors for hypersonic flow measurements, J. of Microelectromech. Syst. 28 (2) (2019) 271-278.

[17] T. Chen, J. Chiu, C. Cheng, M. S. Lu, Design and characterization of capacitively sensed squeeze-film pressure sensors, IEEE Sensors J. 19 (5) (2019) 1653-1660.

[18] H. Yu, X. Yu, Y. Li, Design, fabrication and optimization of a CMOS compatible capacitive pressure sensor, J. of Micromech. and Microeng. 29 (2) (2019) 025009.

[19] K. E. Petersen, Silicon torsional scanning mirror, IBM Journal of Research and Development 24 (5) (1980) 631-637.

[20] S. Afrang, H. Mobki, M. Hassanzadeh, G. Rezazadeh, Design and simulation of a mems analog micro-mirror with improved rotation angle, Microsystem Technologies 25 (3) (2019) 1099-1109.

[21] A. Frangi, A. Guerrieri, N. Boni, R. Carminati, M. Soldo, G. Mendicino, Mode coupling and parametric resonance in electrostatically actuated micromirrors, IEEE Trans. Ind. Electronics 65 (7) (2016) 5962-5969.

[22] H. Hillmer, B. Al-Qargholi, M. M. Khan, N. Worapattrakul, H. Wilke, C. Woidt, A. Tatzel, Optical MEMS-based micromirror arrays for active light steering in smart windows, Japanese Journal of Applied Physics 57 (8S2) (2018) 08PA07.

770

[23] B. Yanhui, J. T. W. Yeow, B. C. Wilson, Design, fabrication, and characteristics of a mems micromirror with sidewall electrodes, J. Microelectromech. Syst. 19 (3) (2010) 619-631. 
[24] L. Ye, G. Zhang, Z. You, Large-aperture khz operating frequency ti-alloy based optical micro scanning mirror for lidar application, Micromachines 8 (4) (2017) 120.

[25] Y. Kusano, Q. Wang, G. Luo, Y. Lu, R. Q. Rudy, R. G. Polcawich, D. A. Horsley, Effects of dc bias tuning on air-coupled pzt piezoelectric micromachined ultrasonic transducers, J. of Microelectromech. Syst. 27 (2) (2018) 296-304.

[26] A. Hajati, D. Latev, D. Gardner, A. Hajati, D. Imai, M. Torrey, M. Schoeppler, Three-dimensional micro electromechanical system piezoelectric ultrasound transducer, Appl. Phys. Lett. 101 (25) (2012) 253101.

[27] S. Mohith, P. N. Karanth, S. Kulkarni, Recent trends in mechanical micropumps and their applications: A review, Mechatronics 60 (2019) $34-$ 55.

[28] A. H. Nayfeh, H. M. Ouakad, F. Najar, S. Choura, E. M. Abdel-Rahman, Nonlinear dynamics of a resonant gas sensor, Nonlinear Dynamics 59 (4) (2010) 607-618.

[29] H. K. Lee, R. Melamud, S. Chandorkar, J. Salvia, S. Yoneoka, T. W. Kenny, Stable operation of mems oscillators far above the critical vibration amplitude in the nonlinear regime, J. of Microelectromech. Syst. 20 (6) (2011) 1228-1230.

[30] A. Gusso, R. L. Viana, A. C. Mathias, I. L. Caldas, Nonlinear dynamics and chaos in micro/nanoelectromechanical beam resonators actuated by two-sided electrodes, Chaos, Solitons and Fractals 122 (2019) 6-16.

[31] S. Baguet, V.-N. Nguyen, C. Grenat, C.-H. Lamarque, R. Dufour, Nonlinear dynamics of micromechanical resonator arrays for mass sensing, Nonlinear Dynamics 95 (2) (2019) 1203-1220. 
[32] P. Taheri-Tehrani, M. Defoort, D. A. Horsley, Synchronization of a micromechanical oscillator in different regimes of electromechanical nonlinearity, Appl. Phys. Lett. 111 (18) (2017) 183503.

[33] A. Garg, S. K. Dwivedy, Nonlinear dynamics of parametrically excited piezoelectric energy harvester with 1:3 internal resonance, Int. J. Nonlin. Mech. 111 (2019) $82-94$.

[34] X. Li, D. Upadrashta, K. Yu, Y. Yang, Analytical modeling and validation of multi-mode piezoelectric energy harvester, Mechanical Systems and Signal Processing 124 (2019) 613 - 631.

[35] G. Gafforelli, A. Corigliano, R. Xu, S.-G. Kim, Experimental verification of a bridge-shaped, nonlinear vibration energy harvester, Appl. Phys. Lett. 105 (20) (2014) 203901.

[36] G. Gafforelli, R. Ardito, A. Corigliano, Improved one-dimensional model of piezoelectric laminates for energy harvesters including three dimensional effects, Composite Structures 127 (2015) 369 - 381.

[37] X. Li, D. Upadrashta, K. Yu, Y. Yang, Analytical modeling and validation of multi-mode piezoelectric energy harvester, Mechanical Systems and Signal Processing 124 (2019) 613 - 631.

[38] H. Sun, M. Yin, W. Wei, J. Li, H. Wang, X. Jin, Mems based energy harvesting for the internet of things: a survey, Microsystem Technologies 24 (7) (2018) 2853-2869.

[39] W. Tian, Z. Ling, W. Yu, J. Shi, A review of mems scale piezoelectric energy harvester, Applied Sciences 8 (4) (2018) 645.

[40] M. Serene, R. Babu, Z. C. Alex, Sensitivity Analysis of Micro-Mass Optical MEMS Sensor for Biomedical IoT Devices, Springer Singapore, Singapore, 2019, pp. 1-12. 
[48] F. M. Alsaleem, M. H. H. Hasan, M. K. Tesfay, A mems nonlinear dynamic approach for neural computing, J. of Microelectromech. Syst. 27 (5) (2018) 780-789.

[49] A. Hajjaj, M. Hafiz, M. Younis, Mode coupling and nonlinear resonances

[41] J. Clark, Self-calibration and performance control of mems with applications for iot, Sensors 18 (12) (2018) 4411.

[42] S. Luo, Q. Sun, W. Cheng, Chaos control of the micro-electro-mechanical resonator by using adaptive dynamic surface technology with extended state observer, AIP Advances 6 (4) (2016) 045104.

[43] L. L. Li, P. M. Polunin, S. Dou, O. Shoshani, B. Scott Strachan, J. S. Jensen, S. W. Shaw, K. L. Turner, Tailoring the nonlinear response of mems resonators using shape optimization, Appl. Phys. Lett. 110 (8) (2017) 081902.

[44] C. Comi, A. Corigliano, V. Zega, S. Zerbini, Non linear response and optimization of a new z-axis resonant micro-accelerometer, Mechatronics 40 (2016) 235-243.

[45] A. Bouchaala, N. Jaber, O. Yassine, O. Shekhah, V. Chernikova, M. Eddaoudi, M. I. Younis, Nonlinear-based mems sensors and active switches for gas detection, Sensors 16 (6) (2016) 758.

[46] M. S. Al-Ghamdi, M. E. Khater, K. M. E. Stewart, A. Alneamy, E. M. Abdel-Rahman, A. Penlidis, Dynamic bifurcation MEMS gas sensors, J. of Micromech. and Microeng. 29 (1) (2018) 015005.

[47] A. Frangi, B. De Masi, F. Confalonieri, S. Zerbini, Threshold shock sensor based on a bistable mechanism: Design, modeling, and measurements, J. of Microelectromech. Syst. 24 (6) (2015) 2019-2026.

of mems arch resonators for bandpass filters, Sci. Rep. 7 (2017) 41820. 
[50] M. H. Hasan, F. M. Alsaleem, H. M. Ouakad, Novel threshold pressure sensors based on nonlinear dynamics of MEMS resonators, J. of Micromech. and Microeng. 28 (6) (2018) 065007.

[51] A. Rezaei Kivi, S. Azizi, A. Khalkhali, Sensitivity enhancement of a mems sensor in nonlinear regime, Intern. J. of Mechanics and Materials in Design 12 (3) (2016) 337-351.

[52] M. A. Hopcroft, W. D. Nix, T. W. Kenny, What is the young's modulus of silicon?, J. of Microelectromech. Syst. 19 (2) (2010) 229-238.

[53] A. Ghisi, F. Fachin, S. Mariani, S. Zerbini, Multi-scale analysis of polysilicon mems sensors subject to accidental drops: Effect of packaging, Microelectronics Reliability 49 (3) (2009) $340-349$.

[54] S. Mariani, R. Martini, A. Ghisi, A. Corigliano, B. Simoni, Monte carlo simulation of micro-cracking in polysilicon mems exposed to shocks, Intern. J. of Fracture 167 (1) (2011) 83-101.

[55] D. I. Son, J. J. Kim, D. I. Kwon, Fracture behavior of single- and polycrystalline silicon films for mems applications, Key Engineering Materials 297-300 (2005) 551-556.

[56] E. D. Reedy, B. L. Boyce, J. W. Foulk, R. V. Field, M. P. de Boer, S. S. Hazra, Predicting fracture in micrometer-scale polycrystalline silicon mems structures, J. of Microelectromech. Syst. 20 (4) (2011) 922-932.

[57] H. Kahn, N. Tayebi, R. Ballarini, R. Mullen, A. Heuer, Fracture toughness of polysilicon mems devices, Sensors and Actuators A: Physical 82 (1) (2000) $274-280$.

[58] F. Najar, A. Nayfeh, E. Abdel-Rahman, S. Choura, S. El-Borgi, Dynam875 ics and global stability of beam-based electrostatic microactuators, J. of Vibration and Control 16 (5) (2010) 721-748. 
[59] X. Xiong, Y. Wu, W. Jone, Material fatigue and reliability of mems accelerometers, in: 2008 IEEE International Symposium on Defect and Fault Tolerance of VLSI Systems, 2008, pp. 314-322.

[67] S. Krylov, N. Dick, Dynamic stability of electrostatically actuated initially curved shallow micro beams, Continuum Mech. Thermodyn. 22 (2010) 445468 .

Vol. 511 of Applied Mechanics and Materials, Trans Tech Publications Ltd, 2014, pp. 565-568.

[62] G. Langfelder, A. Longoni, F. Zaraga, A. Corigliano, A. Ghisi, A. Merassi, A polysilicon test structure for fatigue and fracture testing in micro electro mechanical devices, in: SENSORS, 2008 IEEE, 2008, pp. 94-97.

[63] R. Lifshitz, M. C. Cross, Nonlinear Dynamics of Nanomechanical and Micromechanical Resonators, Wiley-VCH Verlag GmbH \& Co. KGaA, Weinheim, 2009.

[64] S. H. Strogatz, Nonlinear Dynamics And Chaos. Studies in nonlinearity, Sarat Book House, 2007.

[65] H. Cho, B. Jeong, M.-F. Yu, A. F. Vakakis, D. M. McFarland, L. A. Bergman, Nonlinear hardening and softening resonances in micromechanical cantilever-nanotube systems originated from nanoscale geometric nonlinearities, Int. J. Solids and Struct. 49 (2012) 2059-2065.

[66] M. Ganapathia, O. Politb, Dynamic characteristics of curved nanobeams using nonlocal higher-order curved beam theory, Physica E 91 (2017) 190202. 
[68] K.-Q. Pan, J.-Y. Liu, Geometric nonlinear dynamic analysis of curved beams using curved beam element, Acta Mech. Sin. 27 (6) (2011) 10231033.

[69] A. Z. Hajjaj, N. Alcheikh, M. I. Younis, The static and dynamic behavior of mems arch resonators near veering and the impact of initial shapes, Int. J. Nonlin. Mech. 95 (2017) 277-286.

[70] A. Frangi, P. Fedeli, G. Laghi, G. Langfelder, G. Gattere, Near vacuum gas damping in mems: Numerical modeling and experimental validation, J. Microelectromech. Syst. 25 (5) (2016) 890-899.

[71] M. Bao, H. Yang, Squeeze film air damping in mems, Sensors and Actuators A: Physical 136 (1) (2007) 3 - 27.

[72] U. Nabholz, W. Heinzelmann, J. E. Mehner, P. Degenfeld-Schonburg, Amplitude- and gas pressure-dependent nonlinear damping of high-q oscillatory mems micro mirrors, J.of Microelectromech. Syst. 27 (3) (2018) 383-391.

[73] S. Zaitsev, O. Shtempluck, E. Buks, O. Gottlieb, Nonlinear damping in a micromechanical oscillator, Nonlinear Dynamics 67 (1) (2012) 859-883.

[74] L. Lifshitz, M. L. Roukes, Thermoelastic damping in micro- and nanomechanical systems, Phys. Rev. B 61 (2000) 5600-5609.

[75] S. Prabhakar, S. Vengallatore, Thermoelastic damping in hollow and slotted microresonators, J. of Microelectromech. Syst. 18 (3) (2009) 725-735.

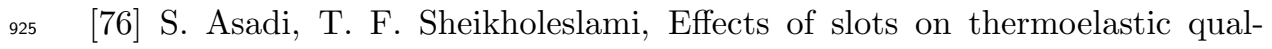
ity factor of a vertical beam mems resonator, Microsystem Technologies 22 (11) (2016) 2723-2730.

[77] R. Abdolvand, H. Johari, G. K. Ho, A. Erbil, F. Ayazi, Quality factor in trench-refilled polysilicon beam resonators, J. Microsyst. Technol. 15 (2006) 471478. 
[78] R. N. Candler, A. Duwel, M. Varghese, S. A. Chandorkar, M. A. Hopcroft, Woo-Tae Park, Bongsang Kim, G. Yama, A. Partridge, M. Lutz, T. W. Kenny, Impact of geometry on thermoelastic dissipation in micromechanical resonant beams, J. of Microelectromech. Syst. 15 (4) (2006) 927-934.

[79] V. Zega, A. Frangi, A. Guercilena, G. Gattere, Analysis of frequency stability and thermoelastic effects for slotted tuning fork mems resonators, Sensors 18 (7) (2018) 2157.

[80] A. Frangi, M. Cremonesi, Semi-analytical and numerical estimates of anchor losses in bistable mems, Int. J. Solids Struct. 9293 (2016) 141148.

[81] J. Rodriguez, S. A. Chandorkar, C. A. Watson, G. M. Glaze, C. H. Ahn, E. J. Ng, Y. Yang, T. W. Kenny, Direct detection of akhiezer damping in a silicon mems resonator, Sci. Rep. 9 (2019) 2045-2322.

[82] S. Adams, F. Bertsch, K. Shaw, N. MacDonald, Independent tuning of linear and nonlinear stiffness coefficients, J. of Microelectromech. Syst. 7 (2) (1998) 172-180.

[83] N. Krakover, S. Krylov, Bistable cantilevers actuated by fringing electrostatic fields, J. of Vibration and Acoustics, Transactions of the ASME $139(4)$.

[84] A. Hariri, J. Zu, R. Ben Mrad, Modeling of wet stiction in microelectromechanical systems (mems), J. of Microelectromech. Syst. 16 (5) (2007) 1276-1285.

[85] R. Ardito, A. Corigliano, A. Frangi, On the analysis of spontaneous adhesion in mems, in: EuroSimE 2009 - 10th International Conference on Thermal, Mechanical and Multi-Physics Simulation and Experiments in Microelectronics and Microsystems, 2009, pp. 1-7.

[86] D. B. Heinz, V. A. Hong, C. H. Ahn, E. J. Ng, Y. Yang, T. W. Kenny, Experimental investigation into stiction forces and dynamic mechanical 
anti-stiction solutions in ultra-clean encapsulated mems devices, J. of Microelectromech. Syst. 25 (3) (2016) 469-478.

[87] A. Guerrieri, A. Frangi, L. Falorni, An investigation on the effects of contact in mems oscillators, J. Microelectromech. Syst. 27 (6) (2018) 963972 .

[88] R. Moorthy, A. Kakodkar, H. Srirangarajan, S. Suryanarayan, An assessment of the newmark method for solving chaotic vibrations of impacting oscillators, Computers and Structures 49 (4) (1993) 597 - 603.

[89] U. Andreaus, L. Placidi, G. Rega, Numerical simulation of the soft contact dynamics of an impacting bilinear oscillator, Communications in Nonlinear Science and Numerical Simulation 15 (9) (2010) 2603 - 2616.

[90] S.-K. Choi, S. T. Noah, Response and stability analysis of piecewiselinear oscillators under multi-forcing frequencies, Nonlinear Dynamics 3 (2) (1992) 105-121.

[91] E. J. Ng, V. A. Hong, Y. Yang, C. H. Ahn, C. L. M. Everhart, T. W. Kenny, Temperature dependence of the elastic constants of doped silicon, J. of Microelectromech. Syst. 24 (3) (2015) 730-741.

[92] A. Jaakkola, M. Prunnila, T. Pensala, J. Dekker, P. Pekko, Determination of doping and temperature-dependent elastic constants of degenerately doped silicon from mems resonators, IEEE Transactions on Ultrasonics, Ferroelectrics, and Frequency Control 61 (7) (2014) 1063-1074.

[93] A. Jaakkola, M. Prunnila, T. Pensala, J. Dekker, P. Pekko, Design rules for temperature compensated degenerately n-type doped silicon mems resonators, J. Microelectromech. Syst. 24 (2015) 1832-1839.

[94] D. Shin, D. Heinz, H.-K. Kwon, Y. Chen, W. Kenny, Lateral diffusion doping of silicon for temperature compensation of mems resonators, in: 2018 IEEE Intern. Symp. on Inertial Sensors and Syst., 2018, pp. 1-4. 
[95] M. Defoort, P. Taheri-Tehrani, D. Horsley, Exploiting nonlinear amplitude-frequency dependence for temperature compensation in silicon micromechanical resonators, Appl. Phys. Lett. 109 (109) (2016) 153502.

[96] A. I. Manevich, L. I. Manevich, The Mechanics of Nonlinear Systems with Internal Resonances, World Scientific, 2005.

[97] C. Chen, D. H. Zanette, D. A. Czaplewski, S. Shaw, D. Lpez, Near vacuum gas damping in mems: Numerical modeling and experimental validation, Nat. Comm. 8 (1) (2017) 15523.

[98] D. A. Czaplewski, C. Chen, D. Lopez, O. Shoshani, A. M. Eriksson, S. Strachan, S. W. Shaw, Bifurcation generated mechanical frequency comb, Phys. Rev. Lett. 121 (2018) 244302.

[99] D. Antonio, D. H. Zanette, D. Lopez, Frequency stabilization in nonlinear micromechanical oscillators, Nat. Comm. 8 (2017) 15523.

[100] A. Hajjaj, F. Alfosail, M. Younis, Two-to-one internal resonance of mems arch resonators, International Journal of Non-Linear Mechanics 107 (2018) $64-72$.

[101] A. Sarrafan, S. Azimi, F. Golnaraghi, B. Bahreyni, A nonlinear rate microsensor utilising internal resonance, Sci. Rep. 9 (2019) 8648.

[102] K. Park, Q. Chen, Y.-C. Lai, Energy enhancement and chaos control in microelectromechanical systems, Phys. Rev. E 77 (2008) 026210.

[103] A. Seleim, S. Towfighian, E. Delande, E. Abdel-Rahman, G. Heppler, Dynamics of a close-loop controlled mems resonator, Nonlinear Dynamics 69 (1) (2012) 615-633.

[104] E. M. Miandoab, A. Yousefi-Koma, H. N. Pishkenari, F. Tajaddodianfar, Study of nonlinear dynamics and chaos in mems/nems resonators, Communications in Nonlinear Science and Numerical Simulation 22 (1) (2015) $611-622$. 
[105] S. Liu, A. Davidson, Q. Lin, Simulation studies on nonlinear dynamics and chaos in a MEMS cantilever control system, J. of Micromech. and Microeng. 14 (7) (2004) 1064-1073.

[106] D. Antonio, D. A. Czaplewski, J. R. Guest, D. López, S. I. Arroyo, D. H. Zanette, Nonlinearity-induced synchronization enhancement in micromechanical oscillators, Phys. Rev. Lett. 114 (2015) 034103.

[107] R. W. Clough, J. Penzien, Dynamics of structures, Mc-Graw Hill, New York, 1993.

[108] J. Rhoads, S. Shaw, K. Turner, Nonlinear dynamics and its applications in micro-and nanoresonators, J. of Dynamic Systems, Measurement and Control, Transactions of the ASME 132 (3) (2010) 1-14.

[109] S. Tiwari, R. N. Candler, Using flexural MEMS to study and exploit nonlinearities: a review, J. of Micromech. and Microeng. 29 (8) (2019) 083002.

[110] T. Veijola, T. Mattila, O. Jakkola, J. Kiihamaki, T. Lamminmaki, A. Oja, K. Ruokonen, H. Sepa, P. Seppala, I. Tittonen, Large-displacement modeling and simulation of micromechanical electrostatically driven resonantors using the harmonic balance method, IEEE MTT-S Int. Microwave Symp. Digest 1 (2000) 99-102.

[111] M. A. Hosen, M. S. H. Chowdhury, M. Y. Ali, A. F. Ismail, An analytical approximation technique for the duffing oscillator based on the energy balance method, Italian J. of pure and applied mathematics 37 (2017) $455-466$.

[112] M. I. Younis, A. H. Nayfeh, A study of the nonlinear response of a resonant microbeam to an electric actuation, Nonlinear Dynamics 31 (1) (2003) 91117.

[113] A. H. Nayfeh, Introduction of perturbation techniques, Wiley, New York, 1981. 
[114] A. H. Nayfeh, D. T. Mook, Nonlinear Oscillations, Wiley Classic Library Edition, 1995.

[115] E. L. Allgower, K. Georg, Numerical Continuation Methods, Springer, 1990.

[116] D. Rugar, P. Grütter, Mechanical parametric amplification and thermo-

[117] W. Zhang, R. Baskaran, K. L. Turner, Effect of cubic nonlinearity on auto-parametrically amplified resonant mems mass sensor, Sensors and Actuators A: Physical 102 (1) (2002) 139 - 150.

[118] M. V. Requa, K. L. Turner, Precise frequency estimation in a micro-

[122] J. F. Rhoads, S. W. Shaw, The impact of nonlinearity on degenerate parametric amplifiers, Appl. Phys. Lett. 96 (23) (2010) 234101.

[123] C. Comi, A. Corigliano, G. Langfelder, A. Longoni, A. Tocchio, B. Simoni, A resonant microaccelerometer with high sensitivity operating in an oselectromechanical parametric resonator, Appl. Phys. Lett. 90 (17) (2007) 173508.

[119] R. Almog, S. Zaitsev, O. Shtempluck, E. Buks, Noise squeezing in a nanomechanical duffing resonator, Phys. Rev. Lett. 98 (2007) 078103.

[120] V. Zega, S. Nitzan, M. Li, C. H. Ahn, E. Ng, V. Hong, Y. Yang, T. Kenny, A. Corigliano, D. A. Horsley, Predicting the closed-loop stability and oscillation amplitude of nonlinear parametrically amplified oscillators, Appl. Phys. Lett. 106 (23) (2015) 233111.

[121] M. Mond, G. Cederbaum, P. B. Khan, Y. Zarmi, Stability analysis of the non-linear mathieu equation, J. of Sound and Vibr. 167 (1) (1993) 77 89.

cillating circuit, J. of Microelectromech. Syst. 19 (5) (2010) 1140-1152. 
[124] M. Aikele, K. Bauer, W. Ficker, F. Neubauer, U. Prechtel, J. Schalk, H. Seidel, Resonant accelerometer with self-test, Sensors and Actuators A: Physical 92 (1) (2001) $161-167$.

[125] H. Ding, W. Wang, B.-F. Ju, J. Xie, A MEMS resonant accelerometer with sensitivity enhancement and adjustment mechanisms, J. of Micromech. and Microeng. 27 (11) (2017) 115010.

[126] A. Tocchio, C. Comi, G. Langfelder, A. Corigliano, A. Longoni, Enhancing the linear range of mems resonators for sensing applications, IEEE Sensors J. 11 (12) (2011) 3202-3210.

[127] J. F. Rhoads, S. W. Shaw, K. L. Turner, J. Moehlis, B. E. DeMartini, W. Zhang, Generalized parametric resonance in electrostatically actuated microelectromechanical oscillators, Journal of Sound and Vibration 296 (4) (2006) $797-829$.

[128] J. Zhang, Y. Su, Q. Shi, A. P. Qiu, Microelectromechanical Resonant Accelerometer Designed with a High Sensitivity, Sensors (Switzerland) 15 (12) (2015) 30293-30310.

[129] J. Zhang, Y. Wang, V. Zega, Y. Su, A. Corigliano, Nonlinear dynamics under varying temperature conditions of the resonating beams of a differential resonant accelerometer, J. of Micromech. and Microeng. 28 (7) (2018) 075004.

[130] R. Melamud, M. Hopcroft, C. Jha, B. Kim, S. Chandorkar, R. Candler, T. W. Kenny, Effects of stress on the temperature coefficient of frequency in double clamped resonators, in: Digest of Technical Papers - International Conference on Solid State Sensors and Actuators and Microsystems, TRANSDUCERS '05, Vol. 1, IEEE, 2005, pp. 392-395.

[131] Y. Zhu, A. Corigliano, H. D. Espinosa, A thermal actuator for nanoscale in situ microscopy testing: design and characterization, J. of micromech. and microeng. 16 (2) (2006) 242. 
[132] C. Comi, A. Corigliano, A. Ghisi, S. Zerbini, A resonant micro accelerometer based on electrostatic stiffness variation, Meccanica 48 (8) (2013) 1893-1900.

[133] A. Caspani, C. Comi, A. Corigliano, G. Langfelder, V. Zega, S. Zerbini, Dynamic nonlinear behavior of torsional resonators in mems, J. Micromech. and Microeng. 24 (2014) 095025 (9pp).

[134] A. Caspani, C. Comi, A. Corigliano, G. Langfelder, V. Zega, S. Zerbini, A differential resonant micro accelerometer for out-of-plane measurements, Proc. Eng. 87 (2014) 640-643.

[135] C. Comi, A. Corigliano, V. Zega, S. Zerbini, Optimal design and nonlinearities in a z-axis resonant accelerometer, in: 16th International Conference on Thermal, Mechanical and Multi-Physics Simulation and Experiments in Microelectronics and Microsystems (EuroSimE), Budapest, Hungary, 2015, pp. 1-6.

[136] L. Ruzziconi, A. H. Ramini, M. I. Younis, S. Lenci, Theoretical prediction of experimental jump and pull-in dynamics in a mems sensor, Sensors 14 (2014) 17089-17111.

[137] G. N. Nielson, G. Barbastathis, Dynamic pull-in of parallel-plate and torsional electrostatic mems actuators, J. Microelectromech. Syst. 15 (2006) $811-821$.

[138] A. H. Nayfeh, M. I. Younis, Dynamic pull-in phenomenon in mems resonators, Nonlinear Dynamics 48 (2007) 153-163.

[139] C. Comi, A. Corigliano, M. Doti, A. Garatti, G. Langfelder, V. Zega, Torsional microresonator in the nonlinear regime: experimental, numerical and analytical characterization, in: Proceedings Eurosensors 2016, Budapest, Hungary, 2016, pp. 1-6.

[140] S. Sonmezoglu, P. Taheri-Tehrani, C. Valzasina, L. G. Falorni, S. Zerbini, S. Nitzan, D. A. Horsley, Single-structure micromachined three-axis gy- 
roscope with reduced drive-force coupling, IEEE Electron Device Letters 36 (9) (2015) 953-956.

[141] V. Zega, G. Langfelder, L. G. Falorni, C. Comi, Hardening, softening, and linear behavior of elastic beams in mems: An analytical approach, J. Microelectomech. Syst. 28 (2) (2019) 189-198.

[142] I. Hotzen, O. Ternyak, S. Shmulevich, D. Elata, Selective stiffening for producing motion conversion mechanisms, Proc. Eng. 87 (2014) 15891592.

[143] I. Hotzen, O. Ternyak, S. Shmulevich, D. Elata, Mass-fabrication compatible mechanism for converting in-plane to out-of-plane motion, in: IEEE International Conference on Micro Electro Mechanical Systems (MEMS), 2015, pp. 897-900.

[144] S. H. Nitzan, V. Zega, M. Li, C. H. Ahn, A. Corigliano, T. W. Kenny, D. A. Horsley, Self-induced parametric amplification arising from nonlinear elastic coupling in a micromechanical resonating disk gyroscope, Sci. Rep. 5 (2015) 9036.

[145] P. Polunin, S. Shaw, Self-induced parametric amplification in ring resonating gyroscopes, Intern. J. of Non-Linear Mechanics 94 (2017) 300 308.

[146] A. B. Sabater, P. D. Swanson, K. M. Moran, Theoretical foundations for scale factor improvement of the disk resonant gyroscope, in: 2017 IEEE Intern. Symp. on Inertial Sensors and Syst., 2017, pp. 1-2.

[147] K. Moran, K. Stanzione, A. Sabater, T. Lewis, V. Badescu, A. Wang, Self-induced parametric amplification in the disk resonant gyroscope, in: 2019 IEEE Intern. Symp. on Inertial Sensors and Syst., 2019, pp. 1-4.

[148] M. H. Matheny, L. G. Villanueva, R. B. Karabalin, J. E. Sader, M. L. Roukes, Nonlinear mode-coupling in nanomechanical systems, Nano Lett. 13 (2013) 1622-1626. 
[149] W. J. Venstra, R. van Leeuwen, H. S. J. van der Zant, Strongly coupled modes in a weakly driven micromechanical resonator, Appl. Phys. Lett. 101 (2012) 243114.

[150] H. J. R. Westra, M. Poot, H. S. J. van der Zant, W. J. Venstra, Nonlinear modal interactions in clamped-clamped mechanical resonators, Phys. Rev. Lett. 105 (2010) 117205. 